\title{
Assessing The Habitat Suitability of Six Coniferous Forests Using Avian Assemblages At High Altitude Ecosystem
}

M. N. Rajpar

Department of Forestry, Shaheed Benazir Bhutto University Sheringal Dir (Upper), Khyber Pakhtunkhwa, Pakistan

S. A. Khan

Department of Forestry, Shaheed Benazir Bhutto University Sheringal Dir (Upper), Khyber Pakhtunkhwa, Pakistan

S. Ullah

Department of Forestry, Shaheed Benazir Bhutto University Sheringal Dir (Upper), Khyber Pakhtunkhwa, Pakistan

A.H. Rajpar

Department of Mechanical Engineering, college of Engineering, Jouf University, Saudi Arabia

M. Zakaria ( $\sim$ mzakaria@upm.edu.my)

Department of Forest Science and Biodiversity, Faculty of Forestry and Environment, Universiti Putra Malaysia

\section{Research Article}

Keywords: birds, conifer forest, density, diversity, habitat, subtropical, vegetation

Posted Date: June 15th, 2021

DOI: https://doi.org/10.21203/rs.3.rs-586649/v1

License: (c) (1) This work is licensed under a Creative Commons Attribution 4.0 International License. Read Full License 


\section{Abstract}

Birds are habitat specialist, i.e. often selects habitat that offers ample services such as a variety of food resources, safe breeding and less disturb sites to satisfy their needs. We aimed to ascertain the conservation status of avian assemblages inhabited in six different coniferous forests. The study employed the point count method to determine the avian assemblages in each forest type. The surveys were conducted employing the distance sampling point count method and determining the floristic composition through direct visual estimation from January 2018 to March 2019 . In total, 2465 bird individuals representing 57 species and 29 families were observed in six coniferous forests. Twelve tree species, nine shrub species, and 14 grass species were identified in the forests. Except for the Streptopelia turtur (vulnerable), all bird species are listed as "least concern" according to the IUCN Red List Category and Criteria 2019-2. Notably, the relative abundance of bird assemblages among six coniferous habitats was significantly different ( $F 5,293=22.8, p<0.05)$. The CAP results reflected that bird diversity indices varied coniferous forests. Strikingly, dry-temperate coniferous forest (BG) was densely populated (5.831 \pm 0.694 birds ha-1) as compared to other habitats. These findings suggested that subtropical coniferous forests are cradles of life for a wide array of avian assemblages and hence should be declared as protected forests to enhance avian assemblages in this region.

\section{Introduction}

Coniferous forests comprise the largest biome on earth, and approximately one-third of the world's coniferous forest areas exist in the northern parts of North America, Europe, and Asia (Spurr and Barnes, 1080; Klappenbach, 2018). They are cradles of life for a wide array of wildlife species, especially birds and mammals (Langat et al., 2016; Duguma et al., 2019). Subtropical coniferous forests are more diverse in terms of their floristic composition (Behera et al., 2002; Bhattarai and Vetass, 2003; Acharya et al., 2011; Gaire et al., 2014) and richness in fauna (Gentili et al., 2015; Ahmed et al., 2018; Mannan et al., 2019). In addition, they provide countless ecosystem services for the livelihood of local communities, i.e., food, raw material for medicine, fuelwood, fodder, fruits, and economic benefits (Rasul, 2014; Maren et al., 2014; Sandhu and Sandhu, 2014; Dhyani and Dhyani, 2016). Pakistan is bestowed with 4.6 million ha of forest area, of which 1.96 million ha form Himalayan coniferous forests (Hasan et al., 2016).

Unfortunately, these unique habitats (coniferous forests) have been poorly protected and have experienced high-level losses and degradation due to human interaction (Wright, 2010). Human footprints, such as timber harvesting, intensive livestock grazing, agriculture expansion, and urbanization, are the most significant drivers of alteration in the habitat structure of the forest (Mahmood, 2003; Wright, 2010; Lawson et al., 2014; Elsen et al., 2017; Mannan et al., 2019). These factors alter the floristic composition and forest structure (Foley et al., 2005; Qasim et al., 2013; Ullah et al., 2016; Mukete et al., 2017), ultimately declining the population of wildlife fauna in subtropical coniferous forests. It has been estimated that, in the past two decades, around $14.0 \%$ of the subtropical forest in the mountains has been lost in low lying areas (Ahmad et al., 2018; Mannan et al., 2018).

Habitat loss and degradation due to human activities pose significant threats to the avian population structure and distribution (Appiah et al., 2009; Hersperger et al., 2010; Feyissa et al., 2014; Mukete et al., 2018). Some species have become threatened and endangered (e.g., rufous-vented prinia - Laticilla burnesii, western tragopan - Tragopan melanocephalus, long-billed warbler - Locustella major, Kashmir flycatcher - Ficedula subrubra, cheer pheasant Catreus wallichii, and black-bellied tern - Sterna acuticauda) across different habitats (i.e., forests, wetlands, highland pastures, foothills, Indus plains, estuaries, deserts, reedbeds, and waterlogged areas) due to deforestation, habitat destruction, and degradation (Puyravaud et al., 2010).

Birds are the best bio-indicators of the coniferous forest ecosystem; i.e., the presence or absence of avian species may indicate the productivity of particular habitats (Waterhouse et al., 2002; Watson et al., 2004; Roberge and Angelstam, 2006). Birds are closely associated with the landscape mosaic, coniferous forest flora and food resources, and reflect the level of disturbance (Jokimaki and Solonen, 2011; Pakkala et al., 2014; Koli, 2014; Menna et al., 2016). The comparison of avian populations among different subtropical coniferous forests has previously been used to determine the conservation status, population trend, habitat preference, and productivity of different habitats (Kattan and Franco, 2004; Harisha and Hosetti, 2009; Khan et al., 2013; Khan and Pant, 2017).

Coniferous forests have been poorly surveyed due to their complex topography, difficult terrain, and harsh weather (Lomolino, 2001). Information about the avian assemblages inhabiting Himalayan subtropical coniferous forests and the detrimental effects on the habitat is scarce. More concrete information about the conservation status and avian assemblages is critically essential to protect and conserve avian species inhabiting the Himalayan subtropical coniferous forests and for better future management planning (Tomialojc and Wesolowski, 2004; Wesolowski et al., 2010). Hence, this study was conducted to examine the population structure of avian species in six subtropical coniferous forests of Pakistan and to identify the habitat suitability. The results will form a foundation for guiding future conservation and protection in the region.

\section{Materials And Methods}

Study Area: The coniferous forests, namely, sub-tropical coniferous forests ( $\mathrm{L} ; 34.89 \mathrm{ha}$ ), sub-tropical coniferous forests (S; $28.25 \mathrm{ha}$ ), dry-temperate coniferous forest ( $Z$; 29.85 ha), dry-temperate coniferous forest (BG; 30.54 ha), mixed dry-temperate coniferous forest (G; 33.78 ha), and mixed drytemperate coniferous forest ( $\mathrm{K} ; 33.77 \mathrm{ha}$ ), occurs in a narrow zone confined within approximately $900-2000$ m elevation above sea level. The vegetation of coniferous forests consists mainly of evergreen cone-bearing and needle-leaved trees, while their multilayered canopy comprises conifers and broadleaved trees, shrubs, and grasses. Each habitat may vary in terms of vegetation species composition and structure. The characteristics of each particular habitat are given in Table 1.

Table 1: Descriptions of each coniferous habitat types 


\begin{tabular}{|c|c|c|c|c|c|c|}
\hline \multirow[t]{2}{*}{ Description } & \multicolumn{6}{|l|}{ Forest Types } \\
\hline & $\begin{array}{l}\text { Sub- } \\
\text { tropical coniferous forests } \\
(\mathrm{L})\end{array}$ & $\begin{array}{l}\text { Sub- } \\
\text { tropical coniferous forests } \\
\text { (S) }\end{array}$ & $\begin{array}{l}\text { Dry- } \\
\text { temperate } \\
\text { coniferous } \\
\text { forest (Z) }\end{array}$ & $\begin{array}{l}\text { Dry- } \\
\text { temperate } \\
\text { coniferous } \\
\text { forest (BG) }\end{array}$ & $\begin{array}{l}\text { Mixed dry- } \\
\text { temperate } \\
\text { coniferous } \\
\text { forest }(\mathrm{G})\end{array}$ & $\begin{array}{l}\text { Mixed dry-temperate coniferous } \\
\text { forest }(\mathrm{K})\end{array}$ \\
\hline Latitude & $\mathrm{N} 34^{0} 47^{\prime} 35^{\prime \prime}$ & N $34^{\circ} 47^{\prime} 35^{\prime \prime}$ & $\begin{array}{l}\mathrm{N} 34^{0} 45^{\prime} \\
01^{\prime \prime}\end{array}$ & N $34^{0} 44^{\prime} 06^{\prime \prime}$ & $\begin{array}{l}\mathrm{N} 34^{0} 42^{\prime} \\
32^{\prime \prime}\end{array}$ & N $34^{0} 42^{\prime} 32^{\prime \prime}$ \\
\hline Longitude & $\mathrm{E} 72^{0} 02^{\prime} 14^{\prime \prime}$ & $\mathrm{E} 72^{0} 02^{\prime} 14^{\prime \prime}$ & $\begin{array}{l}\mathrm{E} 72^{\circ} 02^{\prime} \\
56^{\prime \prime}\end{array}$ & E $72^{0} 06^{\prime} 38^{\prime \prime}$ & E $72^{0} 3^{\prime} 27^{\prime \prime}$ & $\mathrm{E} 72^{0} 3^{\prime} 27^{\prime \prime}$ \\
\hline $\begin{array}{l}\text { Elevation } \\
(\mathrm{m} \text { asl) }\end{array}$ & 788 to 1670 & 997 to 1065 & 965 to 1066 & 1065 to 1170 & 812 to 935 & 964 to 998 \\
\hline $\begin{array}{l}\text { Vegetation } \\
\text { Cover \% }\end{array}$ & $60.0 \%$ & $65.0 \%$ & $75.0 \%$ & $80.0 \%$ & $85.0 \%$ & $80.0 \%$ \\
\hline $\begin{array}{l}\text { Most } \\
\text { Dominant } \\
\text { tree sp. }\end{array}$ & $\begin{array}{l}\text { Pinus roxburghii, Q. } \\
\text { baloot, Mallotus } \\
\text { philippensis, Punica } \\
\text { granatum }\end{array}$ & $\begin{array}{l}\text { P. roxburghii, Q. incana, } \\
\text { Pyrus pashia }\end{array}$ & $\begin{array}{l}\text { P. } \\
\text { wallichiana, } \\
\text { Pinus } \\
\text { roxburghii, } \\
\text { Ailanthus } \\
\text { altissima, } \\
\text { Ficus carica }\end{array}$ & $\begin{array}{l}\text { Pi. } \\
\text { wallichiana, } \\
\text { Morus alba, } \\
\text { Morus nigra, } \\
\text { Acacia } \\
\text { modesta }\end{array}$ & $\begin{array}{l}\text { P. } \\
\text { wallichiana, } \\
P \text {. roxburghii, } \\
\text { Q. delata, } A \text {. } \\
\text { modesta }\end{array}$ & $\begin{array}{l}\text { P. wallichiana, } P \text {, roxburghii, } Q \text {. } \\
\text { baloot, Ailanthus altissima, } F \text {. } \\
\text { carica }\end{array}$ \\
\hline $\begin{array}{l}\text { Most } \\
\text { Dominant } \\
\text { Shrub sp. }\end{array}$ & $\begin{array}{l}\text { Indigofera gerardiana, } \\
\text { Justicia adhatoda, } \\
\text { Ephedra gerardiana }\end{array}$ & $\begin{array}{l}\text { Reptonia muscatencesea, } \\
\text { Berberis lyceum, } \\
\text { Dodonaea viscosa }\end{array}$ & $\begin{array}{l}\text { Solanum } \\
\text { nigrum, } \\
\text { Verbsacum } \\
\text { thapsus, } \\
\text { Ranunculus } \\
\text { muricatus }\end{array}$ & $\begin{array}{l}\text { Berberis } \\
\text { lyceum, } \\
\text { Zanthoxylum } \\
\text { alatum, } \\
\text { Myrtus } \\
\text { communis }\end{array}$ & $\begin{array}{l}\text { Dodonaea } \\
\text { viscosa, } \\
\text { Berberis } \\
\text { lyceum, } \\
\text { Indigofera } \\
\text { gerardiana }\end{array}$ & $\begin{array}{l}\text { Crataegus oxyacantha, marijuar } \\
\text { Dysphania ambrosioides, Tribul, } \\
\text { terrestris }\end{array}$ \\
\hline $\begin{array}{l}\text { Most } \\
\text { Abundant } \\
\text { Grass sp. }\end{array}$ & $\begin{array}{l}\text { Cannabis sativa, Cynodon } \\
\text { dactylon }\end{array}$ & $\begin{array}{l}\text { C. dactylon, Phalaris } \\
\text { minor }\end{array}$ & $\begin{array}{l}\text { Apluda } \\
\text { mutica, } \\
\text { Polygonum } \\
\text { aviculare, } \\
\text { Dichanthium } \\
\text { annulatum }\end{array}$ & $\begin{array}{l}\text { Chrysopogon } \\
\text { montanus, } \\
\text { Polygonum } \\
\text { aviculare, } \\
\text { Rumex } \\
\text { dentatus }\end{array}$ & $\begin{array}{l}\text { C. dactylon, } \\
\text { Heteropogon } \\
\text { contortus }\end{array}$ & $\begin{array}{l}\text { Setaria glauca, Miscanthus } \\
\text { nepalensis }\end{array}$ \\
\hline $\begin{array}{l}\text { Most } \\
\text { Common } \\
\text { herbs }\end{array}$ & $\begin{array}{l}\text { Carthamus oxyacantha, } \\
\text { Verbena officinalis }\end{array}$ & $\begin{array}{l}\text { Chenopodium } \\
\text { ambrosioides, Valeriana } \\
\text { jatamansi }\end{array}$ & $\begin{array}{l}\text { Taraxacum } \\
\text { officinale, } \\
\text { Urtica dioica }\end{array}$ & $\begin{array}{l}\text { Solanum } \\
\text { nigrum, } \\
\text { Stellaria } \\
\text { media }\end{array}$ & $\begin{array}{l}\text { Mentha } \\
\text { longifolia, } \\
\text { Rumex } \\
\text { histatus }\end{array}$ & $\begin{array}{l}\text { Verbena officinali, Rumex } \\
\text { dentatus }\end{array}$ \\
\hline $\begin{array}{l}\text { Surrounding } \\
\text { habitat }\end{array}$ & $\begin{array}{l}\text { Agricultural fields and } \\
\text { human settlements }\end{array}$ & $\begin{array}{l}\text { Agriculture fields and } \\
\text { human settlement }\end{array}$ & $\begin{array}{l}\text { Agriculture } \\
\text { fields and } \\
\text { human } \\
\text { settlement }\end{array}$ & $\begin{array}{l}\text { Agriculture } \\
\text { fields }\end{array}$ & $\begin{array}{l}\text { Agriculture } \\
\text { fields and } \\
\text { human } \\
\text { settlement }\end{array}$ & Agriculture fields and orchards \\
\hline
\end{tabular}

(Note; $\mathrm{L}=$ Laram; $\mathrm{S}$ = Shengari, $\mathrm{Z}$ = Zarback, BG = Baber Gakhai, G = Gulabad and K = Khanpur)

Bird Survey. The distance sampling point count method is one of the most robust and preferred quantitative bird survey methods used for determining the population structure of bird species inhabiting forest habitat (DeSante et al., 2005; Buckland et al., 2008; Gale et al., 2009; Thomas et al., 2010; Ma, 2012). This method involves the visual and auditory detection of birds with fixed or variable radius plots in order to determine avian density (Kissling and Garton, 2006; Meadows et al., 2012). The detection of birds in coniferous forests varies depending on tree density, upper story layer, middle story layer, ground vegetation cover, visibility and sounds of avian species, and observer's skill (Gregory et al., 2007; Melles et al., 2011; Anderson et al., 2015).

Data collection for this study was designed on the basis of (i) habitat structure, i.e., landscape, vegetation richness and density, vegetation complexity, percentage vegetation cover, and vegetation composition (e.g., trees, shrubs, and grasses); (ii) occupancy of cryptic, shy, and skulking species, such as tits, tree creepers, babblers, warblers, and chiffchaffs; (iii) availability and richness of food resources; (iv) human intrusion; and (v) areas best fitted to attract bird species. This is because the vegetation composition and complexity affect the distribution and diversity of species (Souza et al., 2015). Likewise, the availability and distribution of food resources in a particular habitat influence the bird community structure and behavior (Levinton and Kelaher, 2004). Similarly, human encroachment cause alterations in habitat structure, which affects the distribution, diversity, and population of avian species in dwelling habitats (Capucchio et al., 2019).

Bird surveys were completed for selected months, i.e. January 2018 (winter), June and July 2018 (summer), November 2018 (autumn) and March 2019 (spring) across the six coniferous forests using the distance sampling point method. A total of 180 point counts (30 point counts in each habitat, i.e., subtropical coniferous forests (L), sub-tropical coniferous forests (S), dry-temperate coniferous forest (Z), dry-temperate coniferous forest (BG), mixed drytemperate coniferous forest $(\mathrm{G})$ and mixed dry-temperate coniferous forest (K) were collected. The sampling points were selected according to the topography of the study area, the ease of access in rolling terrain, the structure and composition of the vegetation, and the safety of the observer. Point counts within each habitat were separated by $250 \mathrm{~m}$ to minimize the risk of double-counting the same bird individuals (Antongiovanni and Metzer, 2005). Each point was visited at least four times during the 15 consecutive months of the study period. Normally, the bird surveys at the 30 points were completed within $1-5$ days. Efforts were made to cover all six study areas within the same month. Whenever this was not possible, we ensured that the surveys were completed in the following month. 
Only sighted bird species within each point count station sampled were considered. However, some bird species were identified through vocal sound, and their position was determined from where the sound was heard. The birds were surveyed at each point count station for 15 min and allowed a 1 min settling time (Gregory et al., 2004). The radial distance from the bird to the observer was recorded using visual estimation. The birds were surveyed between 7:30 and 11:00 a.m. This time was most suitable to conduct the survey because most of the birds were active in foraging, perching, leafing, and sallying activities. Moreover, to avoid the bias of weather, the survey was stopped immediately when the weather conditions were unfavorable (e.g., rain or thick mist). A radius of 30 meters around the point count was used for the bird survey. All surveys were conducted by the main author.

Vegetation Survey. Assessing the floristic composition (i.e. trees, shrubs, grasses and herbs) are key variables that reflect the territory efficiency, aesthetic beauty, and harbor the assorted bird fauna species meet their needs (Schulz et al., 2009). In each habitat, the assurance of vegetation assemblages, i.e. the percent vegetation cover was done to determine the habitat suitability (Dethier et al., 1993; Hees et al., 2000; Godinez-Alvarez et al., 2009). This is one of the foremost used and accepted methods to inventory the floristic composition in an assortment of forest ecosystems (Mumby et al., 1997; Hudon, 1997; Fernandez-Alaez et al., 2002). The vegetation was inventoried at the same points used for surveying birds Trees, shrubs, grasses, and forbs were identified with the help of a plant taxonomist. Trees were categorized into coniferous and broadleaved. The methodology was followed as described by Yates et al., 2019 and Banag-Moran et al., 2020.

\section{DATA ANALYSIS}

Relative Species Abundance (\%): Relative species plenitude is the number of bird individuals per species that occupied in a coniferous forest habitat. It may change from year, altitude, aspects, weather conditions, geographic range, and restoration endeavors (McGill et al., 2007; Verberk, 2011).

The relative abundance of bird species of coniferous forest was evaluated agreeing to the taking following equation:

Relative Species Abundance $(\%)=I s i / \sum$ Nsi X 100

Where, ISi = Total Number of Individual species, $/ \sum \mathrm{Nsi}=$ Total Number of bird species (Anderson, 2017).

Bird Relative Abundance among Six Coniferous Forests. Bird relative abundance was compared using one-way analysis of variance (ANOVA) and Tukey's honestly significant difference (HSD) test (Analytical Software, version 8.1) by McGraw-Hill (2008).

Diversity Indices: The diversity indices of bird species were analyzed through Community Analysis Package (PCA) Version 4.0 by Henderson and Seaby (2007). In this study, Shannon's diversity index, Margalef's Richness Index, and Pielou J Evenness Index were ascertained to compare the bird diversity indices among six coniferous habitats.

Species Diversity Index. The diversity is a varied and inconstant among the bird assemblages in coniferous forest. It accounts for plenitude by giving data on the irregularity and commonality of bird species. For example; Shannon's diversity index is calculated as follows:

$\mathrm{H}=\sum[(\mathrm{pi}) \times \ln (\mathrm{pi})]$

Where, $\mathrm{H}^{\prime}$ designates diversity, $\mathrm{S}$ indicates the number of species, $i$ specifies the abundance of species, $N$ is the total number of all individuals, $p_{i}$ is the relative abundance of each species, and In is the natural logarithm.

Species Richness Index. It is the number of diverse bird species in a given habitat. It moreover gives data on the homogeneity and irregularity of bird distribution and occurrence. For example; Margalef's Richness Index;

$\mathrm{R}=(\mathrm{S}-1) / \ln \mathrm{N}$

Where, $\mathrm{S}$ is the total number of species and $\mathrm{N}$ is the total number of individuals in the sample.

Species Evenness Index. It is the degree of the relative plenitude of distinctive bird species in a specific range. For example; Pielou J Evenness Index;

$\mathrm{J}=\mathrm{H} / \log (\mathrm{S})$

Where, $\mathrm{H}=$ is the observed Shannon-Wiener index and $\mathrm{S}=$ is the total number of bird species in the coniferous forest.

The correlation between Bird Diversity and Vegetation Structure among Six Coniferous Forests: Pearson's correlation coefficient was applied in order to understand the effects of vegetation structure on bird diversity in the six coniferous forests.

Bird Density among Six Coniferous Forests. Determining an accurate population size is highly important to obtain the current population status of different bird species in a dwelling habitat. The bird population was determined through the DISTANCE Software (Version 7.1) by Buckland et al. (2004).

The key to distance sampling is to use the distribution of observed distances to determine the "detection function" $g(y)$, i.e., the probability of estimating a bird at distance $y$. This function can then be used to ascertain the mean probability of detecting a bird given that it is within $w$ of the point, denoted $P a$. Given an estimate of $\mathrm{Pa}$, bird density can be determined using the following equation:

$\hat{D}=\frac{1}{a} \sum_{i=1}^{n} \frac{1}{\hat{P}_{a}\left(\mathbf{z}_{i}\right)}$ 
where $a$ is the size of the covered region, $n$ is the number of birds detected, and $P^{\wedge} a(\mathbf{z} i)$ is the probability of detecting the $i$-th bird given that it is within $w$ of the point and has the covariate values zi (Buckland et al., 2006; Marques et al., 2007; Fewster et al., 2009).

\section{Results}

Bird Species Composition and Relative Abundance among Six Coniferous Forest: A total of 2465 bird individuals belonging to 53 species and 29 families in six coniferous habitats were identified as; (sub-tropical coniferous forests (L), 50 species, 439 birds, 17.809\%; sub-tropical coniferous forests (S), 48 species, 405 birds, 16.430\%; dry-temperate coniferous forest (BG), 51 species, 447 birds, 18.133\%; dry-temperate coniferous forest (Z), 51 species, 401 birds, 16.267\%; mixed dry-temperate coniferous forest (G), 44 species, 346 birds, 14.036\%; mixed dry-temperate coniferous forest (K), 51 species, 427 birds, $17.322 \%$. Notably, the Turdus atrogularis (sub-tropical coniferous forests (L), $1.623 \%$; sub-tropical coniferous forests (S), $1.217 \%)$, Acridotheres tristis (drytemperate coniferous forest (BG), 1.460\%; mixed dry-temperate coniferous forest (Z), 1.217\%), Turdoides caudata (dry-temperate coniferous forest (BG), $1.298 \%$ ), and Corvus splendens (mixed dry-temperate coniferous forest (L), $2.840 \%$ ) were the most dominant bird species on the basis of the number of detections in the six habitats. In contrast, the Elanus caeruleus (in sub-tropical coniferous forests (L) and dry-temperate coniferous forest (BG), Upupa epops, Copsychus saularis, Accipiter badius (in mixed dry-temperate coniferous forest (BG), Accipiter virgatus (in dry-temperate coniferous forest (Z), Myophonus caeruleus, Columba hodgsonii, Motacilla cinerea, Halcyon smyrnensis (in mixed dry-temperate coniferous forest (L), and Falco tinnunculus (in mixed drytemperate coniferous forest (BG) were the rarest species in the study area (Table 2).

Table 2: Detection of bird species composition and relative abundance among six coniferous forest habitats 


\begin{tabular}{|c|c|c|c|c|c|c|c|c|c|}
\hline \multirow[t]{2}{*}{ Family } & \multirow{2}{*}{$\begin{array}{l}\text { Scientific } \\
\text { Name }\end{array}$} & \multirow{2}{*}{$\begin{array}{l}\text { Common/English } \\
\text { Name }\end{array}$} & \multicolumn{6}{|l|}{ Forest Type } & \multirow{2}{*}{$\begin{array}{l}\text { Conservatio } \\
\text { Status }\end{array}$} \\
\hline & & & $\begin{array}{l}\text { Sub- } \\
\text { tropical } \\
\text { coniferous } \\
\text { forests }(L)\end{array}$ & $\begin{array}{l}\text { Sub- } \\
\text { tropical } \\
\text { coniferous } \\
\text { forests (S) }\end{array}$ & $\begin{array}{l}\text { Dry- } \\
\text { temperate } \\
\text { coniferous } \\
\text { forest } \\
\text { (BG) }\end{array}$ & $\begin{array}{l}\text { Dry- } \\
\text { temperate } \\
\text { coniferous } \\
\text { forest }(Z)\end{array}$ & $\begin{array}{l}\text { Mix Dry- } \\
\text { temperate } \\
\text { coniferous } \\
\text { forest }(\mathrm{G})\end{array}$ & $\begin{array}{l}\text { Mix Dry- } \\
\text { temperate } \\
\text { coniferous } \\
\text { forest (K) }\end{array}$ & \\
\hline Accipitridae & $\begin{array}{l}\text { Accipiter } \\
\text { virgatus }\end{array}$ & Besra & 0.081 & 0.040 & 0.040 & 0.040 & 0 & 0 & LC \\
\hline Upupidae & Upupa epops & Common hoopoe & 0.081 & 0 & 0.040 & 0.081 & 0.081 & 0.081 & LC \\
\hline \multirow[t]{7}{*}{ Columbidae } & $\begin{array}{l}\text { Streptopelia } \\
\text { chinensis }\end{array}$ & Spotted dove & 0.325 & 0.406 & 0.325 & 0.243 & 0.121 & 0.365 & LC \\
\hline & $\begin{array}{l}\text { Streptopelia } \\
\text { senegalensis }\end{array}$ & Laughing dove & 0.243 & 0.162 & 0.365 & 0.203 & 0.162 & 0.162 & LC \\
\hline & $\begin{array}{l}\text { Streptopelia } \\
\text { turtur }\end{array}$ & $\begin{array}{l}\text { European turtle } \\
\text { dove }\end{array}$ & 0.284 & 0.162 & 0.121 & 0.081 & 0.162 & 0.121 & VU \\
\hline & $\begin{array}{l}\text { Streptopelia } \\
\text { orientalis }\end{array}$ & $\begin{array}{l}\text { Oriental turtle } \\
\text { dove }\end{array}$ & 0.365 & 0.203 & 0.243 & 0.284 & 0.243 & 0.162 & LC \\
\hline & Columba livia & Rock dove & 0.121 & 0.162 & 0.406 & 0.365 & 0.203 & 0.406 & LC \\
\hline & $\begin{array}{l}\text { Columba } \\
\text { hodgsonii }\end{array}$ & $\begin{array}{l}\text { Speckled wood } \\
\text { pigeon }\end{array}$ & 0.081 & 0 & 0.081 & 0.162 & 0.040 & 0.081 & LC \\
\hline & $\begin{array}{l}\text { Treron } \\
\text { phoenicoptera }\end{array}$ & $\begin{array}{l}\text { Yellow-footed } \\
\text { green pigeon }\end{array}$ & 0.081 & 0.081 & 0 & 0 & 0.081 & 0 & LC \\
\hline Alcedinidae & $\begin{array}{l}\text { Halcyon } \\
\text { smyrnensis }\end{array}$ & $\begin{array}{l}\text { White-throated } \\
\text { kingfisher }\end{array}$ & 0 & 0.162 & 0.081 & 0.081 & 0.040 & 0.203 & LC \\
\hline Coraciidae & $\begin{array}{l}\text { Coracias } \\
\text { benghalensis }\end{array}$ & Indian roller & 0.162 & 0.081 & 0.081 & 0.081 & 0 & 0.081 & LC \\
\hline Meropidae & $\begin{array}{l}\text { Merops } \\
\text { orientalis }\end{array}$ & Green bee-eater & 0.081 & 0.040 & 0.162 & 0.121 & 0 & 0.081 & LC \\
\hline Falconidae & $\begin{array}{l}\text { Falco } \\
\text { tinnunculus }\end{array}$ & Common kestrel & 0.162 & 0.081 & 0.121 & 0.081 & 0.081 & 0.040 & LC \\
\hline \multirow[t]{2}{*}{ Phasianidae } & $\begin{array}{l}\text { Francolinus } \\
\text { francolinus }\end{array}$ & Black francolin & 0.487 & 0.081 & 0.325 & 0.406 & 0 & 0.325 & LC \\
\hline & $\begin{array}{l}\text { Coturnix } \\
\text { coturnix }\end{array}$ & Common quail & 0.406 & 0.406 & 0.487 & 0.406 & 0.811 & 0.284 & LC \\
\hline Aegithalidae & $\begin{array}{l}\text { Aegithalos } \\
\text { concinnus }\end{array}$ & Black-throated tit & 0.162 & 0 & 0.081 & 0.081 & 0 & 0.121 & LC \\
\hline Alaudidae & $\begin{array}{l}\text { Galerida } \\
\text { cristata }\end{array}$ & Crested lark & 0.081 & 0.162 & 0.243 & 0.162 & 0.203 & 0.325 & LC \\
\hline Campephagidae & $\begin{array}{l}\text { Pericrocotus } \\
\text { flammeus }\end{array}$ & Scarlet minivet & 0.325 & 0.811 & 0.325 & 0.203 & 0.081 & 0.325 & LC \\
\hline Certhiidae & $\begin{array}{l}\text { Certhia } \\
\text { himalayana }\end{array}$ & $\begin{array}{l}\text { Bar-tailed tree } \\
\text { creeper }\end{array}$ & 0.243 & 0.081 & 0.081 & 0.162 & 0 & 0.081 & LC \\
\hline \multirow[t]{4}{*}{ Corvidae } & $\begin{array}{l}\text { Dendrocitta } \\
\text { vagabunda }\end{array}$ & Rufous treepie & 0.081 & 0.162 & 0.243 & 0.325 & 0.081 & 0.081 & LC \\
\hline & $\begin{array}{l}\text { Garrulus } \\
\text { lanceolatus }\end{array}$ & Black-headed jay & 0.203 & 0 & 0.081 & 0.081 & 0 & 0.081 & LC \\
\hline & $\begin{array}{l}\text { Corvus } \\
\text { splendens }\end{array}$ & House crow & 0.284 & 0.730 & 0.487 & 0.568 & 2.840 & 0.121 & LC \\
\hline & $\begin{array}{l}\text { Corvus } \\
\text { macrorhynchos }\end{array}$ & Large-billed crow & 0.406 & 0.081 & 0.162 & 0.243 & 0.081 & 0.162 & LC \\
\hline Dicruridae & $\begin{array}{l}\text { Dicrurus } \\
\text { macrocercus }\end{array}$ & Black drongo & 0.487 & 0.811 & 0.690 & 0.406 & 0.325 & 0.487 & LC \\
\hline Emberizidae & Emberiza cia & Rock bunting & 0.406 & 0.406 & 0.487 & 0.406 & 0.365 & 0.568 & LC \\
\hline Fringillidae & $\begin{array}{l}\text { Carpodacus } \\
\text { erythrinus }\end{array}$ & $\begin{array}{l}\text { Common } \\
\text { rosefinch }\end{array}$ & 1.0142 & 0.527 & 0.811 & 0.406 & 0.487 & 0.811 & LC \\
\hline Hirundinidae & Hirundo rustica & Barn swallow & 0.406 & 0.811 & 1.217 & 0.811 & 0.365 & 0.527 & LC \\
\hline Laniidae & $\begin{array}{l}\text { Lanius } \\
\text { tephronotus }\end{array}$ & $\begin{array}{l}\text { Grey-backed } \\
\text { shrike }\end{array}$ & 0.406 & 0.487 & 0.730 & 0.325 & 0.284 & 0.568 & LC \\
\hline
\end{tabular}




\begin{tabular}{|c|c|c|c|c|c|c|c|c|c|}
\hline & Lanius vittatus & $\begin{array}{l}\text { Bay-backed } \\
\text { shrike }\end{array}$ & 0.162 & 0.121 & 0.284 & 0.081 & 0.121 & 0.162 & LC \\
\hline Leiothrichidae & $\begin{array}{l}\text { Turdoides } \\
\text { caudata }\end{array}$ & Common babbler & 0.811 & 0.609 & 1.258 & 1.298 & 0.406 & 0.690 & LC \\
\hline Leiothrichidae & $\begin{array}{l}\text { Trochalopteron } \\
\text { lineatum }\end{array}$ & $\begin{array}{l}\text { Streaked } \\
\text { laughing thrush }\end{array}$ & 0.284 & 0.406 & 0.325 & 0.487 & 0.243 & 0.609 & LC \\
\hline Monarchidae & $\begin{array}{l}\text { Terpsiphone } \\
\text { paradisi }\end{array}$ & $\begin{array}{l}\text { Indian paradise } \\
\text { flycatcher }\end{array}$ & 0.730 & 0.406 & 0.406 & 0.811 & 0.284 & 0.487 & LC \\
\hline \multirow[t]{2}{*}{ Motacillidae } & Motacilla alba & White wagtail & 0.203 & 0.162 & 0.406 & 0.365 & 0.284 & 0.527 & LC \\
\hline & $\begin{array}{l}\text { Motacilla } \\
\text { cinerea }\end{array}$ & Grey wagtail & 0.081 & 0.162 & 0.162 & 0.081 & 0.040 & 0.243 & LC \\
\hline \multirow[t]{3}{*}{ Muscicapidae } & $\begin{array}{l}\text { Myophonus } \\
\text { caeruleus }\end{array}$ & $\begin{array}{l}\text { Blue whistling } \\
\text { thrush }\end{array}$ & 0.162 & 0.081 & 0.081 & 0.162 & 0.040 & 0.121 & LC \\
\hline & $\begin{array}{l}\text { Copsychus } \\
\text { saularis }\end{array}$ & $\begin{array}{l}\text { Oriental magpie } \\
\text { robin }\end{array}$ & 0 & 0.081 & 0.040 & 0.040 & 0 & 0.121 & LC \\
\hline & $\begin{array}{l}\text { Saxicola } \\
\text { caprata }\end{array}$ & Pied bushchat & 0.121 & 0.081 & 0.121 & 0.162 & 0.243 & 0.162 & LC \\
\hline Oriolidae & Oriolus oriolus & $\begin{array}{l}\text { Eurasian golden } \\
\text { oriole }\end{array}$ & 0.406 & 0.365 & 0.609 & 0.487 & 0.284 & 0.730 & LC \\
\hline Paridae & Parus cinereus & Cinereous tit & 0.771 & 0.446 & 0.365 & 0.406 & 0.162 & 0.487 & LC \\
\hline \multirow[t]{2}{*}{ Passeridae } & $\begin{array}{l}\text { Passer } \\
\text { montanus }\end{array}$ & $\begin{array}{l}\text { Eurasian tree } \\
\text { sparrow }\end{array}$ & 0.609 & 0.811 & 0.406 & 0.527 & 0.487 & 0.730 & LC \\
\hline & $\begin{array}{l}\text { Passer } \\
\text { domesticus }\end{array}$ & House sparrow & 0.811 & 0.892 & 0.730 & 0.487 & 0.406 & 1.0142 & LC \\
\hline \multirow[t]{3}{*}{ Phylloscopidae } & $\begin{array}{l}\text { Phylloscopus } \\
\text { collybita }\end{array}$ & Brown chiffchaff & 0.203 & 0.284 & 0.162 & 0.325 & 0.121 & 0.203 & $\mathrm{LC}$ \\
\hline & $\begin{array}{l}\text { Phylloscopus } \\
\text { chloronotus }\end{array}$ & $\begin{array}{l}\text { Lemon rumped } \\
\text { warbler }\end{array}$ & 0.243 & 0.162 & 0.121 & 0.203 & 0.162 & 0.081 & LC \\
\hline & $\begin{array}{l}\text { Phylloscopus } \\
\text { trochiloides }\end{array}$ & Greenish warbler & 0.284 & 0.081 & 0.162 & 0.121 & 0.203 & 0.243 & LC \\
\hline \multirow[t]{3}{*}{ Pycnonotidae } & $\begin{array}{l}\text { Pycnonotus } \\
\text { cafer }\end{array}$ & $\begin{array}{l}\text { Red-vented } \\
\text { bulbul }\end{array}$ & 0.609 & 0.811 & 0.690 & 0.568 & 0.406 & 0.771 & LC \\
\hline & $\begin{array}{l}\text { Pycnonotus } \\
\text { leucotis }\end{array}$ & $\begin{array}{l}\text { White-eared } \\
\text { bulbul }\end{array}$ & 0.081 & 0.162 & 0.121 & 0.203 & 0.243 & 0.406 & LC \\
\hline & $\begin{array}{l}\text { Pycnonotus } \\
\text { leucogenys }\end{array}$ & $\begin{array}{l}\text { Himalayan } \\
\text { bulbul }\end{array}$ & 0.487 & 0.284 & 0.162 & 0.121 & 0.203 & 0.325 & LC \\
\hline \multirow[t]{3}{*}{ Sturnidae } & $\begin{array}{l}\text { Acridotheres } \\
\text { tristis }\end{array}$ & Common myna & 0.811 & 1.014 & 1.460 & 1.055 & 0.730 & 1.217 & LC \\
\hline & $\begin{array}{l}\text { Sturnia } \\
\text { pagodarum }\end{array}$ & $\begin{array}{l}\text { Brahminy } \\
\text { starling }\end{array}$ & 0.325 & 0.162 & 0.284 & 0.203 & 0.121 & 0.243 & LC \\
\hline & $\begin{array}{l}\text { Sturnus } \\
\text { vulgaris }\end{array}$ & Common starling & 0.487 & 0.406 & 0.609 & 0.568 & 0.811 & 0.568 & LC \\
\hline \multirow[t]{4}{*}{ Turdidae } & $\begin{array}{l}\text { Turdus } \\
\text { atrogularis }\end{array}$ & $\begin{array}{l}\text { Dark-throated } \\
\text { thrush }\end{array}$ & 1.623 & 1.217 & 0.609 & 0.649 & 0.811 & 0.406 & LC \\
\hline & Sub Total & & 439 & 405 & 447 & 401 & 346 & 427 & \\
\hline & \multicolumn{2}{|c|}{ Overall \% in each habitat } & 17.809 & 16.430 & 18.133 & 16.267 & 14.036 & 17.322 & \\
\hline & Grand Total & & & & & 2465 & & & \\
\hline
\end{tabular}

Note: Conservation status determined according to the IUCN Red List, 2019

\section{Comparison of Bird Relative Abundance among Six Coniferous Forests:}

One-way ANOVA and Tukey's HSD test revealed that the bird detections among the six different coniferous forests were significantly different $\left(\mathrm{F}_{5}, 293=22.8, p\right.$ $<0.05$ ). In addition, the relative abundance of avian assemblages was compared through Species and Richness IV (Pisces Software) employing rank abundance curve to determine the species distribution among habitats. The results shown that bird species relative abundance among six coniferous habitats varied (Figure 2)

Comparison of Vegetation Structure and Composition among Six Coniferous Forests. The vegetation inventory results showed that dry-temperate coniferous forest (BG) was more diverse and richer in vegetation composition, i.e., trees (20.98\%; 12 species), shrubs ( $88.88 \% ; 8$ species), grasses ( $92.0 \%$; 3 species) and 
herbs (98.0\%; 7 species), compared to the other habitats. However, vegetation composition varied habitat to habitat depending on soil depth and fertility, intensity of grazing pressure, uncontrolled firewood collection, and land-use change pattern (Table 3).

Table 3. Comparison of vegetation structure and composition among six coniferous forest types. 


\begin{tabular}{|c|c|c|c|c|c|c|c|}
\hline \multirow[t]{2}{*}{ Family } & \multirow[t]{2}{*}{ Scientific Name } & \multicolumn{6}{|l|}{ Forest Type } \\
\hline & & $\begin{array}{l}\text { Sub-tropical } \\
\text { coniferous } \\
\text { forests }(\mathrm{L})\end{array}$ & $\begin{array}{l}\text { Sub-tropical } \\
\text { coniferous } \\
\text { forests (S) }\end{array}$ & $\begin{array}{l}\text { Dry-temperate } \\
\text { coniferous } \\
\text { forest (Z) }\end{array}$ & $\begin{array}{l}\text { Dry-temperate } \\
\text { coniferous } \\
\text { forest (BG) }\end{array}$ & $\begin{array}{l}\text { Mix Dry- } \\
\text { temperate } \\
\text { coniferous forest } \\
\text { (G) }\end{array}$ & $\begin{array}{l}\text { Mix Dry- } \\
\text { temperate } \\
\text { coniferous forest } \\
\text { (K) }\end{array}$ \\
\hline \multicolumn{8}{|l|}{ Tree Species } \\
\hline Pinaceae & $\begin{array}{l}\text { Pinus } \\
\text { roxburghii }\end{array}$ & $6.276 \%$ & $7.221 \%$ & $6.676 \%$ & $8.856 \%$ & $7.493 \%$ & $8.651 \%$ \\
\hline Pinaceae & P. wallichiana & $2.520 \%$ & 0 & 3.951 & $2.929 \%$ & $1.499 \%$ & 0 \\
\hline Fagaceae & $\begin{array}{l}\text { Quercus } \\
\text { delatata }\end{array}$ & $4.223 \%$ & $4.632 \%$ & $5.177 \%$ & $5.245 \%$ & $4.837 \%$ & $3.474 \%$ \\
\hline Rosaceae & Pyrus pashia & $0.136 \%$ & $0.545 \%$ & $0.272 \%$ & $0.136 \%$ & 0 & 0 \\
\hline Lythraceae & $\begin{array}{l}\text { Punica } \\
\text { granatum }\end{array}$ & $0.068 \%$ & $0.885 \%$ & $0.204 \%$ & $0.341 \%$ & 0 & 0 \\
\hline Euphorbiaceae & $\begin{array}{l}\text { Mallotus } \\
\text { phillippensis }\end{array}$ & $0.068 \%$ & $0.613 \%$ & $0.136 \%$ & $0.545 \%$ & $0.272 \%$ & 0 \\
\hline Simaboubaceae & $\begin{array}{l}\text { Ailanthus } \\
\text { altissima }\end{array}$ & 0 & 0 & $0.272 \%$ & $0.341 \%$ & $0.477 \%$ & 0 \\
\hline Moraceae & Ficus carica & $0.204 \%$ & 0 & $0.545 \%$ & $0.409 \%$ & $0.204 \%$ & $0.409 \%$ \\
\hline Moraceae & Morus alba & 0 & $0.545 \%$ & $0.341 \%$ & $0.204 \%$ & 0 & 0 \\
\hline Moraceae & Morus nigra & 0 & 0 & $0.204 \%$ & $0.409 \%$ & 0 & 0 \\
\hline Fabaceae & $\begin{array}{l}\text { Acacia } \\
\text { modesta }\end{array}$ & 0 & 0 & $0.885 \%$ & $1.090 \%$ & $2.929 \%$ & 0 \\
\hline Myrtaceae & $\begin{array}{l}\text { Eucalyptus } \\
\text { camaldulensis }\end{array}$ & $0.613 \%$ & $0.341 \%$ & $0.341 \%$ & $0.477 \%$ & $0.204 \%$ & $0.409 \%$ \\
\hline Sub-Total & & $14.11 \%$ & $14.78 \%$ & $18.974 \%$ & $20.98 \%$ & $17.92 \%$ & $12.94 \%$ \\
\hline \multicolumn{8}{|l|}{ Shrub Species } \\
\hline Fabaceae & $\begin{array}{l}\text { Indigofera } \\
\text { gerardiana }\end{array}$ & 0 & 0 & $32 \%$ & $18 \%$ & $22 \%$ & 0 \\
\hline Rhamnaceae & $\begin{array}{l}\text { Zizihpus } \\
\text { nummularia }\end{array}$ & 0 & 0 & 0 & 0 & 0 & $10 \%$ \\
\hline Acanthaceae & $\begin{array}{l}\text { Justicia } \\
\text { adhatoda }\end{array}$ & $5 \%$ & $18 \%$ & $6 \%$ & $28 \%$ & $8 \%$ & $15 \%$ \\
\hline Sapotaceae & $\begin{array}{l}\text { Reptonia } \\
\text { muscatencesea }\end{array}$ & $12 \%$ & $12 \%$ & 0 & $4 \%$ & 0 & 0 \\
\hline Berberidaceae & $\begin{array}{l}\text { Berberis } \\
\text { lyceum }\end{array}$ & $22 \%$ & 0 & 0 & $31 \%$ & 0 & $23 \%$ \\
\hline sapindaceae & $\begin{array}{l}\text { Dodonaea } \\
\text { viscosa }\end{array}$ & $52 \%$ & $35 \%$ & $48 \%$ & $10 \%$ & $68 \%$ & $43 \%$ \\
\hline Celastraceae & $\begin{array}{l}\text { Gymnosporia } \\
\text { royleana }\end{array}$ & 0 & $30 \%$ & $14 \%$ & $3 \%$ & 0 & $9 \%$ \\
\hline Rutaceae & $\begin{array}{l}\text { Zanthoxylum } \\
\text { alatum }\end{array}$ & $6 \%$ & $5 \%$ & 0 & $2 \%$ & $2 \%$ & 0 \\
\hline Myrtaceae & $\begin{array}{l}\text { Myrtus } \\
\text { communis }\end{array}$ & $3 \%$ & 0 & 0 & $4 \%$ & 0 & 0 \\
\hline Sub-Total & & $66.66 \%$ & $55.55 \%$ & $44.44 \%$ & $88.88 \%$ & $44.44 \%$ & $55.55 \%$ \\
\hline \multicolumn{8}{|l|}{ Grass Species } \\
\hline Poaceae & $\begin{array}{l}\text { Miscanthus } \\
\text { nepalensis }\end{array}$ & $38 \%$ & 0 & 0 & 0 & 0 & 0 \\
\hline Poaceae & $\begin{array}{l}\text { Cynodon } \\
\text { dactylon }\end{array}$ & $24 \%$ & $30 \%$ & $28 \%$ & $34 \%$ & $14 \%$ & $31 \%$ \\
\hline Poaceae & $\begin{array}{l}\text { Heteropogon } \\
\text { contortus }\end{array}$ & $3 \%$ & $5 \%$ & $6 \%$ & $28 \%$ & $10 \%$ & $18 \%$ \\
\hline Poaceae & $\begin{array}{l}\text { Chrysopogon } \\
\text { fulvus }\end{array}$ & $9 \%$ & $7 \%$ & $12 \%$ & $30 \%$ & $8 \%$ & $12 \%$ \\
\hline Sub-Total & & $74.0 \%$ & $42.0 \%$ & $46.0 \%$ & $92.0 \%$ & $32.0 \%$ & $61.0 \%$ \\
\hline
\end{tabular}

Page 9/20 


\begin{tabular}{|c|c|c|c|c|c|c|c|}
\hline \multicolumn{8}{|l|}{ Herbs Species } \\
\hline Asteraceae & $\begin{array}{l}\text { Carthamus } \\
\text { oxyacantha }\end{array}$ & 0 & $28 \%$ & $35 \%$ & $22 \%$ & 0 & $23 \%$ \\
\hline Cannabaceae & $\begin{array}{l}\text { Cannabis } \\
\text { sativa }\end{array}$ & 0 & 0 & $28 \%$ & $25 \%$ & 0 & $39 \%$ \\
\hline Ephedraceae & $\begin{array}{l}\text { Ephedra } \\
\text { gerardiana }\end{array}$ & 0 & 0 & 0 & $7 \%$ & 0 & 0 \\
\hline Asteraceae & $\begin{array}{l}\text { Taraxacum } \\
\text { officinale }\end{array}$ & 0 & $6 \%$ & 0 & $7 \%$ & 0 & 0 \\
\hline Lamiaceae & $\begin{array}{l}\text { Mentha } \\
\text { longifolia }\end{array}$ & 0 & $9 \%$ & 0 & 0 & 0 & 0 \\
\hline Amaranthaceae & $\begin{array}{l}\text { Chenopodium } \\
\text { ambrosioides }\end{array}$ & 0 & $16 \%$ & $18 \%$ & 0 & 0 & $11 \%$ \\
\hline Zygophyllaceae & $\begin{array}{l}\text { Tribulus } \\
\text { terrestris }\end{array}$ & 0 & 0 & $9 \%$ & 0 & 0 & $9 \%$ \\
\hline Solanaceae & $\begin{array}{l}\text { Solanum } \\
\text { nigrum }\end{array}$ & 0 & 0 & 0 & $8 \%$ & $3 \%$ & 0 \\
\hline Scrophuariaceae & $\begin{array}{l}\text { Verbascum } \\
\text { thapsus }\end{array}$ & 0 & 0 & 0 & $5 \%$ & $9 \%$ & 0 \\
\hline Ranunculaceae & $\begin{array}{l}\text { Ranunculus } \\
\text { muricatus }\end{array}$ & 0 & 0 & 0 & $13 \%$ & $4 \%$ & 0 \\
\hline Polygonaceae & $\begin{array}{l}\text { Rumex } \\
\text { dentatus }\end{array}$ & 0 & 0 & 0 & $9 \%$ & $6 \%$ & 0 \\
\hline Polygonaceae & $\begin{array}{l}\text { Polygonum } \\
\text { aviculare }\end{array}$ & 0 & 0 & 0 & 0 & $14 \%$ & 0 \\
\hline Sub-Total & & 0 & $59.0 \%$ & $90.0 \%$ & $96.0 \%$ & $36.0 \%$ & $82.0 \%$ \\
\hline
\end{tabular}

Comparison of Bird Diversity among Six Coniferous Forests. CAP analysis demonstrated that avian diversity, varied among the six coniferous forests. The highest species diversity was documented in mixed dry-temperate coniferous forest $(\mathrm{Z}), \mathrm{H}^{\prime}=3.625 \pm 0.027$ while the lowest was documented in mixed drytemperate coniferous forest $(G), H^{\prime}=3.261 \pm 0.027$. Likewise, dry-temperate coniferous forest (BG) was rich in avian species $\left(R_{1}=8.178 \pm 0.031\right)$ and mixed dry-temperate coniferous forest $(G)$ was the least preferred habitat $\left(R_{1}=7.191 \pm 0.031\right)$. Similarly, bird species were evenly distributed in mixed dry-temperate coniferous forest $(K), E=0.955 \pm 0.006$, and sparsely distributed in mixed dry-temperate coniferous forest $(G), E=0.926 \pm 0.006$ (Table 4).

Table 4: Comparison of bird diversity indices among six coniferous forest habitats.

\begin{tabular}{llll} 
Habitat & Shannon's Index $\left(\mathrm{H}^{\prime}\right)$ & Margalef's Index $\left(\mathrm{R}_{1}\right)$ & Mclntosh's Index $(\mathrm{E})$ \\
\hline Mix Dry-temperate coniferous forest (K) & 3.618 & 7.934 & 0.922 \\
\hline Dry-temperate coniferous forest (BG) & 3.575 & 8.178 & 0.909 \\
\hline Sub-tropical coniferous forests (L) & 3.612 & 7.892 & 0.919 \\
\hline Dry-temperate coniferous forest (Z) & 3.625 & 8.032 & 0.920 \\
\hline Sub-tropical coniferous forests (S) & 3.508 & 7.668 & 0.892 \\
\hline Mix Dry-temperate coniferous forest (G) & 3.261 & 7.191 & 0.829 \\
\hline Overall & 3.641 & 6.405 & 0.926 \\
\hline Standard Error & 0.027 & 0.031 & 0.006
\end{tabular}

Correlation between Bird Diversity and Vegetation Structure among Six Coniferous Forests: Pearson's correlation coefficient (PCC) test revealed that bird diversity had a positive correlation with vegetation structure (trees; $r=0.408, P>0.05$, shrubs; $r=0.138, P>0.05$, and grasses; $r=0.337, P>0.05$ ). However, the level of correlation varied habitats (Table 5).

Table 5: Correlation between bird diversity and vegetation structure (among six coniferous forests). 


\begin{tabular}{llll} 
Habitat Name & $\begin{array}{l}\text { PCC between bird diversity and } \\
\text { trees }\end{array}$ & $\begin{array}{l}\text { PCC between bird diversity and } \\
\text { shrubs }\end{array}$ & $\begin{array}{l}\text { PCC between bird diversity and grasses \& } \\
\text { forbs }\end{array}$ \\
\hline Sub-tropical coniferous forests (L) & $r=0.271, P>0.05$ & $r=0.024, P>0.05$ & $r=0.210, P>0.05$ \\
\hline $\begin{array}{l}\text { Sub-tropical coniferous forests (S) } \\
\text { Dry-temperate coniferous forest (Z) }\end{array}$ & $r=0.193, P>0.05$ & $r=0.013, P>0.05$ & $r=0.027, P>0.05$ \\
\hline $\begin{array}{l}\text { Dry-temperate coniferous forest } \\
\text { (BG) }\end{array}$ & $r=0.318, P>0.05$ & $r=0.138, P>0.05$ & $r=0.251, P>0.05$ \\
\hline $\begin{array}{l}\text { Mix dry-temperate coniferous } \\
\text { forest (G) }\end{array}$ & $r=0.355, P>0.05$ & $r=0.006, P>0.05$ & $r=0.081, P>0.05$ \\
$\begin{array}{l}\text { Mix dry-temperate coniferous } \\
\text { forest (K) }\end{array}$ & $r=0.205, P>0.05$ & $r=0.011, P>0.05$ & $r=0.05$ \\
\hline
\end{tabular}

Comparison of Bird Density among Six Coniferous Forests. Density analysis highlighted that the dry-temperate coniferous forest (BG) was the most densely

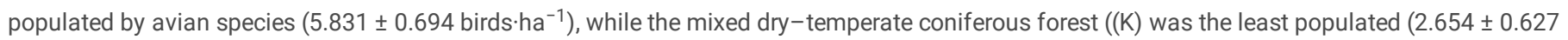
birds'ha-1) (Table 6).

Table 6: Bird density among six coniferous forest habitats.

\begin{tabular}{lll} 
Habitat & Number of Species & Density (birds/ha) \\
\hline Dry-temperate coniferous forest (Z) & 51 & $4.077 \pm 0.362$ \\
\hline Dry-temperate coniferous forest (BG) & 51 & $5.831 \pm 0.694$ \\
\hline Sub-tropical coniferous forests (L) & 50 & $3.187 \pm 0.769$ \\
\hline Mix dry-temperate coniferous forest (G) & 51 & $4.033 \pm 0.987$ \\
\hline Sub-tropical coniferous forests (S) & 48 & $3.152 \pm 0.658$ \\
\hline Mix dry-temperate coniferous forest (K) & 44 & $2.654 \pm 0.627$
\end{tabular}

Furthermore, the density test revealed that mixed dry-temperate coniferous forest (G) harbored the highest population of red-vented bulbul ( $0.952 \pm 0.355$ $\left.\mathrm{ha}^{-1}\right)$, common starling $\left(0.781 \pm 0.261 \mathrm{ha}^{-1}\right)$, and dark-throated thrush $\left(0.589 \pm 0.241 \mathrm{ha}^{-1}\right)$, while mixed dry-temperate coniferous forest $(\mathrm{K})$ featured the highest bird density of Oriolus oriolus $\left(0.848 \pm 0.284 \mathrm{ha}^{-1}\right)$, Dicrurus macrocercus $\left(0.790 \pm 0.283 \mathrm{ha}^{-1}\right)$, Turdus atrogularis $\left(0.769 \pm 0.249\right.$ ha $\left.{ }^{-1}\right)$, Sturnus vulgaris $\left(0.724 \pm 0.174 \mathrm{ha}^{-1}\right)$, and Acridotheres tristis $\left(0.712 \pm 0.217 \mathrm{ha}^{-1}\right)$. Likewise, mixed dry-temperate coniferous forest $(\mathrm{K})$ attracted the higher bird density of Emberiza cia $\left(0.965 \pm 0.471 \mathrm{ha}^{-1}\right)$, Passer montanus $\left(0.842 \pm 0.208 \mathrm{ha}^{-1}\right)$, 0. oriolus $\left(0.790 \pm 0.256 \mathrm{ha}^{-1}\right)$, Turdoides caudata $\left(0.750 \pm 0.112 \mathrm{ha}^{-1}\right)$, and Pycnonotus cafer $\left(0.735 \pm 0.689 \mathrm{ha}^{-1}\right)$. The P. lecogenys $\left(0.985 \pm 0.362 \mathrm{ha}^{-1}\right)$, P. cafer $\left(0.966 \pm 0.320 \mathrm{ha}^{-1}\right)$, Lanius tephronotus $\left(0.915 \pm 0.288 \mathrm{ha}^{-1}\right)$, Parus cinereus $\left(0.796 \pm 0.285 \mathrm{ha}^{-1}\right)$, and Francolinus francolinus $\left(0.793 \pm 0.370 \mathrm{ha}^{-1}\right)$ densely occupied the sub-tropical coniferous forests $(\mathrm{L})$ habitat. Similarly, sub-tropical coniferous forests (S) was more populated by the Indian paradise flycatcher $\left(0.992 \pm 0.291 \mathrm{ha}^{-1}\right), 0$. oriolus $\left(0.926 \pm 0.292 \mathrm{ha}^{-1}\right), P$. cinereus $\left(0.844 \pm 0.214 \mathrm{ha}^{-1}\right)$, Emberiza cia $\left(0.793 \pm 0.224 \mathrm{ha}^{-1}\right)$, and $D$. macrocercus $\left(0.772 \pm 0.276 \mathrm{ha}^{-1}\right)$, while dry-temperate coniferous forest $(\mathrm{Z})$ was more populated by the Trochalopteron lineatum $\left(0.896 \pm 0.385 \mathrm{ha}^{-1}\right)$, P. cafer $\left(0.867 \pm 0.295 \mathrm{ha}^{-1}\right)$, Terpsiphone paradisi $\left(0.841 \pm 0.302 \mathrm{ha} \mathrm{a}^{-1}\right)$, Motacilla alba $\left(0.783 \pm 0.125 \mathrm{ha}^{-1}\right)$, and $F$. francolinus $\left(0.726 \pm 0.289 \mathrm{ha}^{-1}\right)$. However, the densities of 19 species in mixed dry-temperate coniferous forest (Z), 20 species in dry-temperate coniferous forest (BG), 21 species in mixed dry-temperate coniferous forest (K), 17 species in sub-tropical coniferous forests (G), 25 species in sub-tropical coniferous forests (S), and two species in dry-temperate coniferous forest $(\mathrm{L})$ were not determined due to a low number of bird detections, i.e., fewer than five detections (Table 7).

Table 7: Comparison of bird density (birds' ha-1) among six coniferous forest habitats. 


\begin{tabular}{|c|c|c|c|c|c|c|c|}
\hline \multirow[t]{3}{*}{ Family } & \multirow{3}{*}{$\begin{array}{l}\text { Scientific } \\
\text { Name }\end{array}$} & \multicolumn{6}{|c|}{ Name of Forest Type } \\
\hline & & $\begin{array}{l}\text { Mixed dry- } \\
\text { temperate } \\
\text { coniferous forest } \\
\text { (G) }\end{array}$ & $\begin{array}{l}\text { Dry-temperate } \\
\text { coniferous } \\
\text { forest (BG) }\end{array}$ & $\begin{array}{l}\text { Mixed dry- } \\
\text { temperate } \\
\text { coniferous forest } \\
(\mathrm{K})\end{array}$ & $\begin{array}{l}\text { Sub-tropical } \\
\text { coniferous } \\
\text { forests (L) }\end{array}$ & $\begin{array}{l}\text { Sub-tropical } \\
\text { coniferous } \\
\text { forests (S) }\end{array}$ & $\begin{array}{l}\text { Dry-temperate } \\
\text { coniferous } \\
\text { forest }(\mathrm{Z})\end{array}$ \\
\hline & & \multicolumn{6}{|l|}{ Density (birds/ha) } \\
\hline \multirow[t]{3}{*}{ Accipitridae } & $\begin{array}{l}\text { Accipiter } \\
\text { virgatus }\end{array}$ & - & - & - & - & - & - \\
\hline & $\begin{array}{l}\text { Elanus } \\
\text { caeruleus }\end{array}$ & - & - & - & - & - & - \\
\hline & $\begin{array}{l}\text { Accipiter } \\
\text { badius }\end{array}$ & - & - & - & - & - & - \\
\hline Upupidae & Upupa epops & - & - & - & - & - & - \\
\hline \multirow[t]{7}{*}{ Columbidae } & $\begin{array}{l}\text { Streptopelia } \\
\text { chinensis }\end{array}$ & - & $0.261 \pm 0.102$ & $0.622 \pm 0.142$ & $0.399 \pm 0.210$ & - & $0.677 \pm 0.251$ \\
\hline & $\begin{array}{l}\text { Streptopelia } \\
\text { senegalensis }\end{array}$ & - & $0.246 \pm 0.164$ & - & $0.272 \pm 0.104$ & - & $0.318 \pm 0.235$ \\
\hline & $\begin{array}{l}\text { Streptopelia } \\
\text { turtur }\end{array}$ & - & - & - & $0.328 \pm 0.108$ & - & - \\
\hline & $\begin{array}{l}\text { Streptopelia } \\
\text { orientalis }\end{array}$ & $0.278 \pm 0.144$ & $0.285 \pm 0.132$ & - & - & $0.115 \pm 0.082$ & $0.274 \pm 0.123$ \\
\hline & Columba livia & $0.234 \pm 0.140$ & $0.486 \pm 0.252$ & $0.313 \pm 0.813$ & - & - & $0.567 \pm 0.234$ \\
\hline & $\begin{array}{l}\text { Columba } \\
\text { hodgsonii }\end{array}$ & - & - & - & - & $0.285 \pm 0.124$ & - \\
\hline & $\begin{array}{l}\text { Treron } \\
\text { phoenicoptera }\end{array}$ & - & - & - & - & - & - \\
\hline Alcedinidae & $\begin{array}{l}\text { Halcyon } \\
\text { smyrnensis }\end{array}$ & - & - & $0.142 \pm 0.114$ & - & - & - \\
\hline Coraciidae & $\begin{array}{l}\text { Coracias } \\
\text { benghalensis }\end{array}$ & - & - & - & - & - & - \\
\hline Meropidae & $\begin{array}{l}\text { Merops } \\
\text { orientalis }\end{array}$ & - & - & - & - & - & - \\
\hline Falconidae & $\begin{array}{l}\text { Falco } \\
\text { tinnunculus }\end{array}$ & - & - & - & - & - & - \\
\hline \multirow[t]{2}{*}{ Phasianidae } & $\begin{array}{l}\text { Francolinus } \\
\text { francolinus }\end{array}$ & - & $0.356 \pm 0.274$ & $0.244 \pm 0.164$ & $0.793 \pm 0.370$ & - & $0.726 \pm 0.289$ \\
\hline & $\begin{array}{l}\text { Coturnix } \\
\text { coturnix }\end{array}$ & $0.204 \pm 0.111$ & $0.350 \pm 0.189$ & $0.326 \pm 0.140$ & $0.440 \pm 0.174$ & $0.136 \pm 0.042$ & $0.142 \pm 0.484$ \\
\hline Aegithalidae & $\begin{array}{l}\text { Aegithalos } \\
\text { concinnus }\end{array}$ & - & - & - & - & - & - \\
\hline Alaudidae & $\begin{array}{l}\text { Galerida } \\
\text { cristata }\end{array}$ & $0.216 \pm 0.086$ & - & $0.494 \pm 0.126$ & - & - & $0.198 \pm 0.086$ \\
\hline Campephagidae & $\begin{array}{l}\text { Pericrocotus } \\
\text { flammeus }\end{array}$ & - & $0.225 \pm 0.148$ & $0.465 \pm 0.146$ & $0.410 \pm 0.189$ & $0.674 \pm 0.231$ & $0.257 \pm 0.142$ \\
\hline Certhiidae & $\begin{array}{l}\text { Certhia } \\
\text { himalayana }\end{array}$ & - & - & - & $0.278 \pm 0.144$ & - & - \\
\hline \multirow[t]{4}{*}{ Corvidae } & $\begin{array}{l}\text { Dendrocitta } \\
\text { vagabunda }\end{array}$ & - & $0.428 \pm 0.248$ & - & - & - & $0.145 \pm 0.077$ \\
\hline & $\begin{array}{l}\text { Garrulus } \\
\text { lanceolatus }\end{array}$ & - & - & - & - & - & - \\
\hline & $\begin{array}{l}\text { Corvus } \\
\text { splendens }\end{array}$ & $0.563 \pm 0.445$ & $0.263 \pm 0.163$ & - & $0.320 \pm 0.210$ & $0.379 \pm 0.119$ & $0.487 \pm 0.213$ \\
\hline & $\begin{array}{l}\text { Corvus } \\
\text { macrorhynchos }\end{array}$ & - & $0.293 \pm 0.180$ & - & $0.721 \pm 0.251$ & - & - \\
\hline Dicruridae & $\begin{array}{l}\text { Dicrurus } \\
\text { macrocercus }\end{array}$ & $0.399 \pm 0.210$ & $0.790 \pm 0.283$ & $0.315 \pm 0.514$ & $0.166 \pm 0.552$ & $0.772 \pm 0.276$ & $0.148 \pm 0.548$ \\
\hline Emberizidae & Emberiza cia & $0.410 \pm 0.189$ & $0.663 \pm 0.128$ & $0.965 \pm 0.471$ & $0.490 \pm 0.191$ & $0.793 \pm 0.224$ & $0.205 \pm 0.655$ \\
\hline Fringillidae & Carpodacus & $0.254 \pm 0.106$ & $0.252 \pm 0.142$ & $0.515 \pm 0.703$ & $0.607 \pm 0.251$ & $0.450 \pm 0.231$ & $0.174 \pm 0.697$ \\
\hline
\end{tabular}

Page 12/20 
erythrinus

\begin{tabular}{|c|c|c|c|c|c|c|c|}
\hline Hirundinidae & Hirundo rustica & $0.431 \pm 0.112$ & $0.260 \pm 0.180$ & $0.373 \pm 0.210$ & $0.158 \pm 0.466$ & $0.333 \pm 0.118$ & $0.219 \pm 0.109$ \\
\hline \multirow[t]{2}{*}{ Laniidae } & $\begin{array}{l}\text { Lanius } \\
\text { tephronotus }\end{array}$ & $0.296 \pm 0.049$ & $0.464 \pm 0.198$ & $0.480 \pm 0.566$ & $0.915 \pm 0.288$ & $0.295 \pm 0.137$ & $0.106 \pm 0.085$ \\
\hline & Lanius vittatus & - & - & - & - & - & $0.260 \pm 0.129$ \\
\hline Leiothrichidae & $\begin{array}{l}\text { Turdoides } \\
\text { caudata }\end{array}$ & $0.264 \pm 0.103$ & $0.574 \pm 0.139$ & $0.750 \pm 0.112$ & $0.507 \pm 0.234$ & $0.714 \pm 0.241$ & $0.229 \pm 0.876$ \\
\hline Leiothrichidae & $\begin{array}{l}\text { Trochalopteron } \\
\text { lineatum }\end{array}$ & $0.282 \pm 0.120$ & $0.413 \pm 0.136$ & $0.415 \pm 0.584$ & $0.296 \pm 0.149$ & $0.672 \pm 0.291$ & $0.896 \pm 0.385$ \\
\hline Monarchidae & $\begin{array}{l}\text { Terpsiphone } \\
\text { paradisi }\end{array}$ & $0.346 \pm 0.142$ & $0.680 \pm 0.126$ & $0.283 \pm 0.110$ & $0.145 \pm 0.536$ & $0.992 \pm 0.291$ & $0.841 \pm 0.302$ \\
\hline \multirow[t]{2}{*}{ Motacillidae } & Motacilla alba & $0.320 \pm 0.210$ & $0.572 \pm 0.272$ & $0.685 \pm 0.642$ & - & - & $0.783 \pm 0.125$ \\
\hline & $\begin{array}{l}\text { Motacilla } \\
\text { cinerea }\end{array}$ & - & - & $0.168 \pm 0.102$ & - & - & - \\
\hline \multirow[t]{3}{*}{ Muscicapidae } & $\begin{array}{l}\text { Myophonus } \\
\text { caeruleus }\end{array}$ & - & - & - & $0.346 \pm 0.142$ & - & - \\
\hline & $\begin{array}{l}\text { Copsychus } \\
\text { saularis }\end{array}$ & - & - & - & $0.431 \pm 0.112$ & - & - \\
\hline & $\begin{array}{l}\text { Saxicola } \\
\text { caprata }\end{array}$ & $0.245 \pm 0.129$ & - & - & $0.966 \pm 0.320$ & - & - \\
\hline Oriolidae & Oriolus oriolus & $0.328 \pm 0.108$ & $0.848 \pm 0.284$ & $0.790 \pm 0.256$ & $0.651 \pm 0.217$ & $0.926 \pm 0.292$ & $0.246 \pm 0.182$ \\
\hline Paridae & Parus cinereus & - & $0.574 \pm 0.214$ & $0.366 \pm 0.384$ & - & $0.844 \pm 0.214$ & $0.133 \pm 0.411$ \\
\hline \multirow[t]{2}{*}{ Passeridae } & $\begin{array}{l}\text { Passer } \\
\text { montanus }\end{array}$ & $0.291 \pm 0.124$ & $0.210 \pm 0.168$ & $0.842 \pm 0.208$ & $0.267 \pm 0.100$ & $0.460 \pm 0.094$ & $0.243 \pm 0.116$ \\
\hline & $\begin{array}{l}\text { Passer } \\
\text { domesticus }\end{array}$ & $0.231 \pm 0.115$ & $0.341 \pm 0.149$ & $0.608 \pm 0.101$ & $0.648 \pm 0.226$ & $0.587 \pm 0.149$ & $0.119 \pm 0.404$ \\
\hline \multirow[t]{3}{*}{ Phylloscopidae } & $\begin{array}{l}\text { Phylloscopus } \\
\text { collybita }\end{array}$ & - & $0.396 \pm 0.264$ & $0.261 \pm 0.122$ & $0.796 \pm 0.285$ & $0.129 \pm 0.094$ & - \\
\hline & $\begin{array}{l}\text { Phylloscopus } \\
\text { chloronotus }\end{array}$ & - & $0.192 \pm 0.104$ & - & $0.245 \pm 0.12$ & - & - \\
\hline & $\begin{array}{l}\text { Phylloscopus } \\
\text { trochiloides }\end{array}$ & $0.131 \pm 0.112$ & - & $0.282 \pm 0.124$ & $0.282 \pm 0.120$ & - & - \\
\hline \multirow[t]{3}{*}{ Pycnonotidae } & $\begin{array}{l}\text { Pycnonotus } \\
\text { cafer }\end{array}$ & $0.952 \pm 0.355$ & $0.219 \pm 0.128$ & $0.735 \pm 0.689$ & $0.245 \pm 0.129$ & $0.694 \pm 0.210$ & $0.867 \pm 0.295$ \\
\hline & $\begin{array}{l}\text { Pycnonotus } \\
\text { leucotis }\end{array}$ & $0.272 \pm 0.104$ & $0.135 \pm 0.147$ & $0.585 \pm 0.597$ & - & - & - \\
\hline & $\begin{array}{l}\text { Pycnonotus } \\
\text { leucogenys }\end{array}$ & $0.167 \pm 0.134$ & - & $0.386 \pm 0.136$ & $0.985 \pm 0.362$ & $0.128 \pm 0.102$ & - \\
\hline \multirow[t]{3}{*}{ Sturnidae } & $\begin{array}{l}\text { Acridotheres } \\
\text { tristis }\end{array}$ & $0.170 \pm 0.987$ & $0.712 \pm 0.217$ & $0.658 \pm 0.108$ & $0.463 \pm 0.159$ & $0.716 \pm 0.185$ & $0.178 \pm 0.723$ \\
\hline & $\begin{array}{l}\text { Sturnia } \\
\text { pagodarum }\end{array}$ & - & $0.206 \pm 0.121$ & $0.296 \pm 0.148$ & $0.167 \pm 0.134$ & - & $0.234 \pm 0.096$ \\
\hline & $\begin{array}{l}\text { Sturnus } \\
\text { vulgaris }\end{array}$ & $0.781 \pm 0.261$ & $0.724 \pm 0.174$ & $0.415 \pm 0.719$ & $0.632 \pm 0.193$ & $0.476 \pm 0.270$ & $0.173 \pm 0.572$ \\
\hline Turdidae & $\begin{array}{l}\text { Turdus } \\
\text { atrogularis }\end{array}$ & $0.589 \pm 0.241$ & $0.769 \pm 0.249$ & $0.254 \pm 0.113$ & $0.462 \pm 0.110$ & $0.582 \pm 0.283$ & $0.232 \pm 0.916$ \\
\hline
\end{tabular}

\section{Discussions}

Subtropical, dry-temperate and mixed dry-temperate coniferous forests are known as the most productive habitats harboring a higher avian assemblage of endemic and threatened species (Joshi et al., 2012). They are diverse in a topography supporting luxuriant (thick and healthy) vegetation that forms mosaics and provides vital resources for a wide array of avian species (Myers et al., 2000; Elsen, et al., 2017; Elsen et al., 2018). Ascertaining the bird population parameters, vegetation structure, and food resources is crucial for indicating the habitat suitability and productivity of a forest ecosystem (Karubian et al., 2005; Cassey et al., 2007; Amar et al., 2008; IUCN, 2011). Furthermore, the vegetation structure and composition could help our understanding the bird species diversity pattern (Vetaas and Grytnes, 2002; Rahbek, 2005; Grytnes and McCain, 2007).

Most bird species are habitat specialists, i.e., they utilize different layers of vegetation for foraging, shelter, and breeding purposes. Some avian species prefer the forest floor (e.g., common babblers, common quails, common hoopoe, magpie robins, blue whistling thrush, and pied bushchat), while others utilize the 
tree canopy, i.e., Indian paradise flycatchers, scarlet minivets, golden orioles, cinereous tits, yellow-footed green pigeon, speckled wood pigeon, streaked laughing thrush, and common rosefinch (Gardner et al., 2009; Malhi et al., 2014).

Notably, the food resources among the six coniferous forests are not distributed uniformly and may vary from area to area, depending on topography, microclimate, altitude, and vegetation structure and composition. Likewise, the bird relative abundance among these six forests also varies. The highest relative abundance was detected in dry-temperate coniferous forest (BG) and the lowest was detected in mix dry-temperate coniferous forest (G). This result indicated that the birds often select an area on the basis of proximate factors, i.e., landscape, terrain, substrate, microclimate, characteristics, extent of the disturbance, predation, and occurrence of food resources. These factors determine avian community parameters such as diversity, density, and distribution. Another reason could be that the floristic diversity shaping the forest structure influenced the bird population structure. Blake (2007) reported that the bird relative abundance and species composition might vary due to vegetation structure and complexity.

The "highest" bird density was estimated in the dry-temperate coniferous forest (BG) as compared to the other habitats. The higher bird density in drytemperate coniferous forest (BG) was due to the occurrence of fruiting trees (e.g., Ficus carica, Morus alba, and M. nigra), shrubs (i.e., Zizihpus nummularia, Reptonia muscatencesea, Berberis lyceum, Gymnosporia royleana, Zanthoxylum alatum, and Myrtus communis), grasses, Solanum nigrum, and flowering trees (i.e., Acacia modesta and Ailanthus altissima) that provide adequate food sources to harbor the denser avian population. The dark-throated thrush, blue whistling thrush, and streaked laughing thrush prefer shrubs in their search for insects, arachnids, and berries. Likewise, the red-vented bulbul, Himalayan bulbul, and white-eared bulbul often utilizes fruiting trees and shrubs for berries and grasses for insects (Brooks, 2013; Old et al., 2014; Barnagaud et al., 2014; Thibault et al., 2018).

As well, the dry-temperate coniferous forest (BG) was rich in bird species. The richness was due to the complex structure and composition of the vegetation, which attracted the greatest richness of the avian species. For instance, trees that carry nuts ( $P$. wallichiana), juicy and fleshy fruits $(M$. alba, $M$. nigra, $A$. modesta), shrubs that carries the berries (Z. alatum, S. nigrum,), plums (B. lyceum and $M$. communis), grasses bears flowers (Verbascum thapsus, Ranunculus acris, $S$. media) and herbs that carry cereals ( $P$. aviculare, $R$. dentatus, $C$. montanus, and Heteropogon contortus). It was previously reported that bird species often prefer to use a complex habitat that is more diverse in vegetation composition and rich in food resources (Wu et al., 2013; Joshi and Bhatt, 2015; Saha et al., 2016). Likewise, Casas et al. (2016) stated that the vegetation structure and composition offer ideal weather conditions, adequate food resources (fruits and insects), and suitable breeding sites to harbor a wide array of avian populations to perform multiple activities.

Foraging guild results demonstrated that Indian paradise flycatchers selected pine trees for perching after sallying. During sallying, they catch insects while flying (Gokula and Vijayan, 2003; Rassussen and Anderton, 2012; Das and Adhikari, 2019), such as flies, bugs, beetles, spiders, moths, butterflies, and damselflies. The scarlet minivet prefers the tree crown for foraging and perching. It was observed that minivets flushed insects such as flies, moths, grasshoppers, crickets, cicadas, and caterpillars in foliage through gleaning in the crown and caught them on while flying. Likewise, the Eurasian golden oriole and common starlings used common fig, wax apple, and barberry to consume a wide array of fruits and berries, and prey on insects, especially caterpillars. The wax apple populated degraded habitats such as forest edges and mixed vegetation for foraging, particularly on insects (including dragonflies, honeybees, grasshoppers, moths, beetles, and crickets), through sallying, gleaning, and hovering (Somasundaram and Vjayan, 2008). They mainly utilized forest edges and cultivated areas to forage on a variety of food items, i.e., fruits, lizards, rodents, and large insects.

Notably, the black francolin, common quail, and bay-backed shrike were confined to forest edges, shrubs, and grasses, as these are a shy species with secretive behavior. Another reason may be that the forest edges provide cover from predators (Fuisz and Yosef, 1998; Pande et al., 2004; Antczak et al., 2005). Furthermore, the white-throated kingfisher selected riparian habitats and forest edges for perching and foraging (Asokan and Ali, 2010; Coulombe et al., 2011; Kesler, 2012). Moreover, other birds, for instance, the greenish warbler, preferred to use conifer trees for foraging. During foraging, they glean in the crown to prey on midges, caterpillars, beetles, leafhoppers, bugs, wasps, moths, and spiders. However, sometimes, these birds sail in dense shrubs to catch insects on their wing (Johnson and Sherry, 2001). Likewise, the bar-tailed tree creeper also selected coniferous trees for foraging. Upon reaching the top of the conifer through progressive hops, they then fly down to the base of the next tree trunk and start climbing again in a spiral fashion in their search for food (i.e., insects, caterpillars, and spiders).

Apparently, the speckled wood pigeon and rock pigeon were concentrated in fruiting trees and shrubs (i.e., common fig, wind prickly ash, yellow Himalayan raspberry, and barberry) to forage for berries and fleshy fruits. Likewise, the European turtle dove, oriental turtle dove, and spotted dove preferred grounds to feed on grains and seeds of grasses (e.g., pine woods drop seed, beard grass, milkweed, and wild sunflower). The variation in habitat selection and food utilization showed that habitat structure, land-use pattern, and food resources are the major driving factors that significantly influence the habitat use and distribution of avian species (Casas et al., 2016). It could be that the heterogeneity in vegetation composition enables the formation of complex habitats (Hu et al., 2018), which harbor a higher avian diversity (Jankowski et al., 2009; Kissling et al., 2010; Paudel and Vetaas, 2014).

\section{Conclusions}

Based on the findings of this study, dry-temperate coniferous forest (BG) is rich in vegetation species composition and represents a highly productive habitat for a variety of avian species, compared to the other coniferous forests. The occurrence of birds reflects the forest stand structure, site productivity (food resources), vegetation composition, and distribution pattern. Furthermore, it was observed that the vegetation composition and food resources are the major driving factors affecting the habitat selection and home range of avian species. Hence, special attention should be given to the dry-temperate coniferous forest (BG) in order to maintain its high quality and productivity, which are essential for future conservation efforts aimed at harboring a high avian population. This could be that, it is dominated by broadleaf tree species; Ficus carica, Morus Alba, M. nigra, Acacia modesta and Eucalyptus camaldulensis and shrub vegetation, i.e., Reptonia muscatencesea, Berberis lyceum, Dodonaea viscosa, Zanthoxylum alatum and Myrtus communis that offer suitable foraging and safe hideout for avian species. Furthermore, the understorey vegetation is comprised of Solanum nigrum, Verbascum thapsus, Ranunculus 
muricatus, Polygonum aviculare Rumex dentatus and grasses like Cynodon dactylon, Heteropogon contortus, Chrysopogon fulvus and Miscanthusnepalensis that bear a variety of grains and attracted a variety of insects that is a staple diet of avian species. Furthermore, going forward, the remaining coniferous forests can be declared as protected habitats to enhance their healthy avian population.

\section{Abbreviations}

IUCN = International Union for Conservation of Nature, CAP = Community Analysis Package, BG = Baber Gakhai, L $=\mathrm{Laram}, \mathrm{S}=\mathrm{Shengari,} Z=\mathrm{Z}$ Zarback, $\mathrm{G}=$ Gulabad, $\mathrm{K}=$ Khanpur, LC = Least Concern, VU = Vulnerable, $\mathrm{N}=$ North, $\mathrm{E}=\mathrm{East}, \mathrm{m}=$ Meter, asl = Above sea level and ANOVA = Analysis of Variance

\section{Declarations}

Consent for Publication: We are agreed to publish entitle "Assessing the habitat suitability of six coniferous forests using avian assemblages at high altitude ecosystem" manuscript under BMC Ecology and Evolution.

Availability of data and material: All data and material is available

Competing Interest: There are no competing interests among authors

Funding: This research was funded by Universiti Putra Malaysia Grant No: UPM-9638000

\section{Authors' Contribution:}

a) Rajpar, M.N.: Designed the study and wrote the manuscript

b) Khan, S.A.: Collected and analyzed the data

c) Ullah, S. Prepared the map and help in data analysis

d) Rajpar, A.H.: help in data analysis and English editing

e) Zakaria, M.: Reviewed and edited the manuscript

Acknowledgments: The authors acknowledge the Universiti Putra Malaysia for financial support and the Department of Wildlife Timergrah, Khyber Pakhtunkhwa for for granting the permission to conduct this valuable research work.

\section{References}

1. Acharya, B.K., Chettri, B. and Vijayan, L., 2011. Distribution pattern of trees along an elevation gradient of eastern Himalaya, India. Acta Oecologica, 37: 329-336.

2. Ahmad, A., Liu, Q.I.J., Nizami, S.M., Mannan, A. and Saeed, S., 2018. Carbon emission from deforestation, forest degradation and wood harvest in the temperate region of Hindukush Himalaya, Pakistan between 1994 and 2016. Land Use Pol., 78: 781-790.

3. Amar, A., Amidon, F., Arroyo, B., Esselstyn, J.A. and Marshall, A.P., 2008. Population trends of the forest bird community on Pacific island of Roto, Mariana Island. Condor, 110: 421-427.

4. Anderson, A.S., Marques, T.A., Shoo, L.P. and Williams, S.E., 2015. Detectability in audio-visual surveys of tropical rainforest birds: The influence of species, weather and habitat characteristics. PLoS One, 10(6): E0128464.

5. Anderson, V. 2017. Criteria for evaluating quantitative research. Human Resource Development Quarterly, 28(2): 125-133.

6. Antczak, M., Harmada, M. and Tryjanowski, P., 2005. Spatio-temporal changes in Great Grey Shrike Lanius excubitor impaling behavior from food catching to communication signs. Ardea, 93(1): 101-107.

7. Antongiovanni, M. and Metzer, J.P., 2005. Influence of matrix habitats on the occurrence of insectivorous bird species in Amazonian forest fragments. Biological Conservation, 122(3): 441-451.

8. Appiah, M., Blay, D., Damnyag, L., Dwomoh, F.K., Pappinen, A. and Luukkanen, O., 2009. Dependence on forest resources and tropical deforestation in Ghana. Environ. Dev. Sustain., 11: 471-487.

9. Asokan, S. and Ali, A.M.S., 2010. Foraging behavior of selected insectivorous birds in Cauvery Delta region of Nagapattinam District, Tamil Nadu, India. Journal of Threatened Taxa, 2(2): 690-694.

10. Banag-Moran, C., Bautista, F.A., Bonifacio, K.A.M., De Guzman, C.A.M.L., Lim, J.L., Tandang, D.N. and Dagamac, N.H.A. 2020. Variations in floristic composition and community structure between disturbed and undisturbed lowland forest in Aklan, Philippines. Geology, Ecology and Landscapes, URL: https://doi.org/10.1080.24749508.2020.1814187.

11. Barnagaud, J.Y., Barbaro, L., Papix, J., Deconchat, M. and Brocker, E.G., 2014. Habitat filtering by landscape and local forest composition in native and exotic New Zealand birds. Ecology, 95(1): 78-87.

12. Behera, M.D., Kushwaha, S.P.S. and Roy, P.S., 2002. High plant endemism in an Indian hotspot eastern Himalaya. Biodiversity and Conservation, 11: 669682. 
13. Bhattari, K.R. and Vetaas, O.R., 2003. Variation in plant species richness of different life forms along a subtropical elevation gradient in the Himalayas, east Nepal. Global Ecology and Biogeography, 12: 327-340.

14. Blake, J.G., 2007. Neotropical forest bird communities: a comparison of species richness and composition at local and regional scales. The Condor, 109: 237-255

15. Brooks, D.M., 2013. Ecology, behavior, and reproduction of an introduced population of Red-vented bulbuls (Pycnonotus cafer) in Houston, Texas. Wilson J. Ornithol., 125(4): 800-808.

16. Buckland, S.T., Anderson, D.R., Burnhan, K.P., Lake, J.L., Borchers, D.L. and Thomas, L., 2004. Advance Distance Sampling; Estimating Abundance of Biological Populations. London; Campman and Hall. Pp. 141-172. ISBN: 0-19-850927-8.

17. Buckland, S.T., Mardsen, S.J. and Green, R.E., 2008. Estimating bird abundance: making methods work. Bird Conservation International, 18 : S91-S108.

18. Buckland, S.T., Summers, R.W., Borchers, D.L. and Thomas, L. 2006. Point transect sampling with traps or lures. Journal of Applied Ecology, 43 , 377-384.

19. Capucchio, M.T., Colombino, E., Tarantola, M., Biagini, D., Alborali, L.G., Maisano, A.M., Scali, F., Raspa, F., Valle, E., Biasato, I., Schiavone, A., Solgni, C., Bar, V., Gili, C. and Guarda, F. 2019. The disturbed habitat and its effects on the animal population, habitat of the world-biodiversity and threat, Carmelo Maria Musarella, Ana Cano Ortiz and Ricardo Guinto Canas. IntechOpen. DOI: 10.5772/intechopen.84872.

20. Casas, G., Darski, B., Ferreira, P.M.A., Kindel, A. and Muller, S.C., 2016. Habitat structure influences the diversity, richness and composition of bird assemblages in successional Atlantic rainforests. Tropical Conservation Science, 9(1): 503-524.

21. Cassey, P., Craig, J.L., McArdle, B.H. and Barraclough, R.K., 2007. Distance sampling techniques compared for a New Zealand endemic passerine (Philesturnus carunculatus rufusater). New Zealand Journal of Ecology, 31: 223-231.

22. Coulombe, G.L., Kesler, D.C. and Gouni, A., 2011. Agricultural coconut forest as habitat for the critically endangered Tuamotu Kingfisher (Tordiramphus gambieri gertrudae). Auk, 128(2): 283-292.

23. Das, N. and Adhikari, S., 2019. Study of nesting behavior of Asian Paradise Flycatcher Terpsiphone paradise (Aves: Passeriformes: Monorchidae) from southern West Bengal, India. Journal of Threatened Taxa, 11(6): 13782-13785.

24. DeSante. D.F., Sillett, T.S., Siegel, R.B., Saracco, J.F., Romo, C.A., Morales, S., Cerezo, A., Kaschube, D., Mila, B. and Grosselet, M., 2005. Assessing habitatspecific overwintering survival of Neotropical migratory land birds. Pp. 926-936 in: Bird Conservation Implementation and Integration in the Americas (CJ Ralph, D Rich, Eds.). USDA Forest Service General Technical Report. PSW-GTR-191.

25. Dethier, M.N., Graham, E.S., Cohen, S. and Tear, L.M., 1993. Visual versus random point percent cover estimation: 'objective' is not always better. Mari. Ecol. Prog. Series, 96: 93-100.

26. Dhyani, S. and Dhyani, D., 2016. Significance of provisioning ecosystem services from moist temperate forest ecosystem: lesson from upper Kedarnath valley, Garwal, India. Energy, Ecology and Environment, 1: 109-121.

27. Duguma, L.A., Atela, J., Minang, P.A., Ayana, A.N., Gizachew, B., Nzyoka, J.M. and Bernard, F., 2019. Deforestation and forest degradation as environmental behavior: unpacking realities shaping community actions. Land, 8: 26; DOI: 10.3390/land8020026

28. Elsen, P.R., Kalyanaraman, R., Ramesh, K. and Wilcove, D.S., 2017. The importance of agricultural land for Himalayan birds in winter. Conservation Biology, 31(2): 416-426.

29. Elsen, P.R., Ramesh, K. and Wilcove, D.S. (2018). Conserving Himalayan birds in highly seasonal forested and agricultural landscapes. Conservation Biology, 32(6): 1313-1324

30. Fewster, R.M., Buckland, S.T., Burnham, K.P., Borchers, D.L., Jupp, P.E., Laake, J.L. and Thomas, L. 2009. Estimating the encounter rate variance in distance sampling. Biometrics, 65, 225-236.

31. Feyissa, A.K., Suryabhagavan, K.V. and Satishkumar, B., 2014. Assessment of habitat changes in Holeta watershed Central Oromia, Ethiopia. Int. J. Earth Sci. Eng., 7: 1370-1375.

32. Foley, R.D., Asner, C., Barford, G., Bonan, S.R., Carpenter, F.S., Chapin, M.T., Coe, G.D., Gibbs, J.H., Helkowski, T., Holloway, E.A., Kucharik, C.J., Monfreda, J.A., Prentice, N. and Snyder, P.K., 2005. Global consequences of land use. Science, 309: 570-574.

33. Fuisz, T.I. and Yosef, R., 1998. Habitat choice of post-breeding Red-backed Shrike (Lanius colluriro) in northeastern Hungary. IBCE Tech. Publ., 7: 2629.

34. Gaire, N.P., Koirala, M., Bhuju, D.R., Borgaonkar, H.P., 2014. Treeline dynamics with climate change at the central Nepal Himalaya. Climate of the Past, 10: 1277-1290.

35. Gale, G.A., Round, P.D., Pierce, A.J., Nimuan, S., Pattanavibool, A. and Brookelman, W.Y., 2009. A field-test of distance sampling methods for a tropical forest bird community. Auk, 126: 439-448.

36. Gardner, T.A., Barlow, J., Chazdon, R., Ewers, R.M., Harvey, C.A., Peres, C.A. and Sodhi, N.S., 2009. Prospects for tropical forest biodiversity in a humanmodified world. Ecol. Lett., 12: 561-582.

37. Gentili, R., Badola, H.K. and Birks, H.J.B., 2015. Alpine biodiversity and refugia in a changing climate. Biodiversity, 16: 193-195.

38. Godinez-Alvarez, H., Herrick, J.E., Mattocks, M., Toledo, O. and Van Zee, J., 2009. Comparison of three vegetation monitoring methods: their relative utility for ecological assessment and monitoring. Ecological Indicators, 9: 1001-1008.

39. Gokula, V. and Vijayan, L., 2003. Foraging and nesting behavior of Asian Paradise Flycatcher Terpsiphone paradise in Mudumalai wildlife sanctuary, Tamil Nadu, India. Forktail, 19: 142-144.

40. Gregory, R.D., Gibbons, D.W., Donald, P.F., 2004. Bird census and survey techniques. In: Sutherland, W.J., Newton, I., Green, R.E. (Eds.), Bird Ecology and Conservation. A Handbook of Techniques. Oxford University Press, Oxford, pp. 17-55. 
41. Gregory, R.D., Vorisek, P., Strien, A.V., Melying, A.W.G., Jiguet, F., Fornasri, L., Rief, J., Chylarecki, P. and Burfield, I.J., 2007. Population trends of widespread birds in Europe. Ibis, 149(2): 78-97.

42. Grimmett, R., Inskipp, C. and Inskipp, T., 2011. Birds of Indian Subcontinent. Illustrate Edition. Christopher Helm. ISBN: 978-1408127636. Pp. 528.

43. Harisha, M.N. and Hosetti, B.B. 2009. Diversity distribution of avifauna of Lakkavalli range forest, Bhadra Wildlife Sancturay, Western Ghat, India. Ecoprint, 16: $21-27$.

44. Hassan, M.A., Tarar, M.A., Arshad, M.I., Gulshan, A.B. and Iqbal, M.A. 2016. Determinants and consequences of forestation in the Indus River belt area of Dera Ghazi Khan, Pakistan. Journal of Environment and Earth Science, 6(9): 35-43.

45. Hees, V., Willem, W.S. and Bert, R.M., 2000. Ocular estimates of understory vegetation structure in closed Picea glauca/ Betula papyrifera forest. J. Vege. Sci., 11: 195-200.

46. Henderson, P.A. and Seaby, R.M.H., 2007. Community Analysis Package 4.0, Pisces Conservation Ltd, Lymington, UK.

47. Hersperger, A.M., Gennaio, M.P., Verburg, P.H. and Burgi, M., 2010. Linking land change with driving forces and actors: four conceptual models. Ecol. Soc., 15(4): 1-17.

48. Hu, Y., Ding, Z., Jiang, Z., Quan, Q., Guo, K., Tian, L., Hu, H. and Gibson, L., 2018. Birds in the Himalays: What drives beta diversity patters along an elevational gradient? Ecology and Evolution, 8(23): 11704-11716.

49. IUCN. 2011. Relist of threatened species. Version 2011.2.www.iucnredlist.org. Accessed 5 February 2012.

50. Jankowski, J.E., Ciecka, A.L., Meyer, N.Y. and Rabenold, K.N., 2009. Beta diversity along environmental gradients: implications of habitat specialization in tropical montane landscapes. J. Animal Ecology, 78: 315-327.

51. Johnson, M.D. and Sherry, T.W., 2001. Effects of food availability on the distribution of migratory warblers among habitats in Jamaica. Journal of Animal Ecology, 70(4): 546-560.

52. Jokimaki, J., and Solonen, T., 2011. Habitat association of old forest bird species in managed boreal forests characterized by forest inventory data. Ornis Fennica, 88: 57-70.

53. Joshi, K. and Bhatt, D., 2015. Avian species distribution along elevation at Doon valley (foot hills of western Himalayas), Uttarakhand and its association with vegetation structure. J. Asia-Pacific Biod., 8: 158-167.

54. Joshi, K.K., Bhatt, D. and Thapliyal, A., 2012. Avian diversity and its association with vegetation structure in different elevation zones of Nainital district (Western Himalayan) of Uttarkhand. Int. J. Biodiver. Conserv., 4(11): 364-376.

55. Karubian, J., Fabara, J., Yunes, D., Jorgenson, J.P., Romo, D. and Smith, T.B., 2005. Temporal and spatial patterns of Macaw abundance in the Ecuadorian Amazon. Condor, 107: 617-626.

56. Kattan, G.H. and Franco, P., 2004. Bird diversity along elevation gradients in the Andes of Columbia: area and mass effects. Global Ecology and Biogeography, 13: 451-458.

57. Kesler, D.C., 2012. Foraging habitat distribution affect territory size and shape in Tuamotu Kingfisher. Intern. J. Zool., Article ID: 632969, P. 7. https.//doi.org/10.1155/2012/632969.

58. Khan, M.S. and Pant, A., 2017. Conservation status, species composition, and distribution of avian community in Bhimbandah Wildlife Sanctuary, India. Journal of Asia-Pacific Biodiversity, 10: 20-26.

59. Khan, M.S., Aftab, A., Nawab, S.A., Ilyas, O. and Khan, A., 2013. Composition and comparison status of avian species at Hastinapur Wildlife Sanctuary, Uttar Pradesh, India. Journal of Threatened Taxa, 5: 4714-4721.

60. Kissling, M.L. and Garton, E.O., 2006. Estimating detection probability and density from point-count surveys: A combination of distance and doubleobserver sampling. The Auk, 123(3): 735-752.

61. Kissling, W.D., Field, R., Korntheuer, H., Heyde, U. and Bohning-Gaese, K., 2010. Woody plants and the prediction of climate-change impacts on bird diversity. Phil. Trans. Roy. Soci. B: Biol. Scie., 365L 2035-2045.

62. Klappenbach, L. 2018. Explore fascinating facts about forest biome. Web Accessed on $9^{\text {th }}$ November, 2019 at URL: https://www.thoughtco.com/overviewof-the-forest-biome-130162?print.

63. Koli, V.K. 2014. Diversity and status of avifauna in Todgarh-Raoli Wildlife Sanctuary, Rajasthan, India. Journal of Asia-Pacific Biodiversity, 7: $401-407$.

64. Langat, D.K., Maranga, E.K. and Aboud, A.A., 2016. Cheboiwo, J.K. Role of forest resources to local livelihoods: The case of East Mau Forest ecosystem, Kenya. Intern. J. Forest. Resear., 4537354. http://dx.doi.org/10.1155/2016/4537354

65. Lawson, S., Blundell, A., Cabarle, B., Basik, N., Jenkins, M. and Canby, K., 2014. Consumer Goods and Deforestation: An Analysis of the Extent and Nature of Illegality in Forest Conversion for Agriculture and Timber Plantations. Forest Trend Report Series, Washington, DC, USA.

66. Levinton, J., and Kelaher, B., 2004. Opposing organizing forces of deposit-feeding marine communities. J. Exp. Mar. Biol. Ecol., 300: 65-8210.

67. Lomolino, M., 2001. Elevation gradients of species density: historical and prospective views. Global Ecology and Biogeography, 10: 3-13

68. Ma, X., 2012. Distance sampling to estimate the abundance of birds with sector and radial radar detection methods. Procedia Environmental Sciences, 13: 2284-2303.

69. Mahmood, I. 2003. Deforestation in NWFP, NIPA Journal, 8(5): 75-101.

70. Malhi, Y., Gardner, T.A., Goldsmith, G.R., Silman, M.R. and Zelazowski, P., 2014. Tropical forests in the Anthropocene. Annu. Rev. Envir. Resour, 39: 125159.

71. Mannan, A., Liu, J., Zhongke, F., Khan, T.U., Saeed, S., Mukete, B., ChaoYong, S., Yongxiang, F., Ahmed, A., Amir, M., Ahmad, S. and Shah, S., 2019. Application of land-use/land cover changes in monitoring and projecting forest biomass carbon loss in Pakistan, Global Ecology and Conservation, 17:

Page $17 / 20$ 
e00535.

72. Mannan, A., Zhongke, F., Khan, U.T., Saeed, S., Amir, M., Khan, M.A., Badshah, M.T., 2018. Variation in tree biomass and carbon stocks with respect to attitudinal gradient in the Himalayan forest of northern Pakistan. J. Oure. Algebra, 4(1): 21-28.

73. Maren, I.E., Bhattarai, K.R. and Chaudhary, R.P., 2014. Forest ecosystem services and biodiversity in contrasting Himalayan forest management system. Environmental Conservation, 41: 73-83.

74. Marques, T.A., Thomas, L., Fancy, S.G. and Buckland, S.T. 2007. Improving estimates of bird density using multiple covariate distance sampling. The Auk, $127,1229-1243$.

75. McGill, B.J., Etienne, R.S., Gray, J.S., Alonso, D., Anderson, M.J., Benecha, H.K., Dornelas, M., Equist, B.J., Green, J.L., He, F., Hulbert, A.H., Magurran, A.E., Marquet, P.A., Maurer, B.A., Ostling, A., Soykan, C.U., Ugland, K.I., White, E.P., 2007. Species abundance distributions: moving beyond single prediction theories to integration within an ecological framework. Ecol. Lett., 10(10: 995 - 1015.

76. McGraw-Hill, C. 2008. Statistix 8.1; Analytical Software. Tallahassee, Florida Maurice/Thomas text. ISBN: 0073402818.

77. Meadows, S., Moller, H. and Weller, F., 2012. Reduction of bias when estimating bird abundance within small habitat fragments. New Zealand Journal of Ecology, 36: 408-415.

78. Melles, S.J., Fortin, M.J., Lindsay, K. and Badzinski, D., 2011. Expanding northward: influence of climate change, forest connectivity and population processes on a threatened species range shift. Global Change Biology, 17: 17-31.

79. Menna, M., Maazi, M.C., Telailia, S., Saheb, M., Boutabia, L., Chafrour, A. and Houhamdi, M., 2016. Richness and habitat relationships of forest birds in the Zeen Oak woodland (Forest of Bounezrarne, South-Ahras). Pakistan J, Zool., 48(4): 1059-1069.

80. Mukete, B., Sun, Y., Baninla, Y., Achem, B., Bakia, M., Sajjad, S. and Chalwe, P., 2017. Perspective of remote sensing and gis application in tropical forest management. Amer. J. Agric. Fores., 5(3): 33-39.

81. Mukete, B., Sun, Y., Daniel, E., Sajjad, S. and Mannan, A., 2018. Assessing the drivers of land use change in rumpi hills forest protected area, Cameroon. J. Sustain. For., 37(6): 592-618.

82. Myers, N., Mittermier, R.A., Mittermier, C.G., da Fonseca, G.A.B and Kent, J., 2000. Biodiversity hotspots for conservation priorities. Nature, $40: 853-858$.

83. Old, J.M., Spencer, R.J. and Wolfenden, J., 2014. The common myna (Sturnus tristis) in urban, rural and semi-ruralareas in Greater Sydney and its surrounds. Emu, 114(3): 241-248.

84. Pakkala, T., Linden, A., Titainen, J., Tomppo, E. and Kouki, J., 2014. Indicators of forest biodiversity: which bird species predict high breeding bird assemblage diversity in boreal forest at multiple spatial scales? Ann. Zool. Fennici, 51: 457-476

85. Pande, S., Pawshe, A., Santi, N. and Mahbal, A., 2004. Status, habitat preferences and population estimates of non-breeding shrikes Lanius spp. In Maharashtra and Karnataka states, India. Biological Letters, 41(2): 65-69.

86. Paudel, S. and Vetaas, O.R., 2014. Effects of topography and land use on woody plant species composition and beta diversity an arid Trans-Himalayan landscape, Nepal. J. Mountain Science, 11: 1112-1122

87. Puyravaud, J-P., Davidar, P. and Laurance, W.F., 2010. Cryptic destruction of India's native forest. Conservation Letters, 3: $390-394$.

88. Qasim, M., Hubacek, K., Termansen, M. and Fleskens, L. 2013. Modeling land use change across elevation gradients in district Swat, Pakistan. Reg. Environ. Change, 13(3): 567-581.

89. Rahbek, C., 2005. The role of spatial scale and the precipitation of large-scale species richness patterns. Ecology Letters, 8: 224-239.

90. Rassussen, P.C. and Anderton, J.C., 2012. Birds of South Asia: The Riple Guide, $2^{\text {nd }}$ Volume, II Edition. Smithosnian Institution, Michigan State University and Lynx Edicions, Washington DC. Pp. 688.

91. Rasul, G., 2014. Food, water, and energy security in south Asia: A nexus perspective from the Hindu Kush Himalayan region. Environ. Sci. Poli., 39: $35-48$.

92. Roberge, J-M. and Angelstam, P., 2006. Indicator species among resident forest birds - across-regional evaluation in northern Europe. Biological Conservation, 130: 134-147.

93. Robson, C., 2011. Field Guide to the Birds of South-east Asia. Third Edition. New Holland Australia. ISBN: 978-1780090498. Pp. 544.

94. Saha, S., Rajwar, G.S. and Kumar, M., 2009. Forest structure, diversity and regeneration potential along altitudinal gradient in Dhanauliti of Garhwal Himalaya. Forest System, 25(2): e058.

95. Sandhu, H. and Sandhu, S., 2014. Linking ecosystem services with the constituents of human well-being for poverty alleviation in eastern Himalayas. Ecological Economics, 107: 65-75.

96. Schulz, B.K., Bechtold, W.A., Zarnoch, S.J., 2009. Sampling and estimation procedures for vegetation diversity and structure indicator. Gen. Tech. Rep. PNW-GTR-781. Department of Agriculture, Forest services, Pacific Northwest research station. Pp. 53.

97. Somasundaram, S. and Vijayan, L., 2008. Foraging behavior and guild structure of birds in the Montane wet temperate forest of the Palni Hills, South India. Podoces, 3: 79-91.

98. Souza, A.M., Fogaca, F.N.O, Cunico, A.M. and Higuti, J. 2015. Does the habitat structure controls the distribution and diversity of the odonatofauna? Braz. J. Biol., 75(3): 598-606.

99. Spurr, S.H. and Barnes, B.V., 1980. Forest Ecology. John Wiley and Sons, New York.

100. Thibault, M., Vidal, E., Potter, M.A. and Sanchez, T.F., 2018. The invasive Red-vented bulbul (Pycnonotus cafer) outcompetes native birds in a tropical biodiversity hotspot. PLOSONE, 13(2): e0192249.

101. Thomas, L., Buckland, S.T., Rexstad, E.A., Laake, J.L., Strindberg, S., Hedley, S.L., Bishop, J.R.B., Marques, T.A. and Burnham, K.P., 2010. Distance software: design and analysis of distance sampling surveys for estimating population size. Journal of Applied Ecology, 47: 5-14. 
102. Tomialojc, L. and Wesolowski, T., 2004. Diversity of Bialowieza forest avifauna in space and time. Journal of Ornithology, 145: 81-92.

103. Ullah, S., Farooq, M., Shafique, M., Siyab, M.A., Kareem, F. and Dees, M., 2016. Spatial assessment of forest cover and land use changes in the Hindukush mountain ranges of northern Pakistan. J. Mt. Sci., 13(7): 1229-1237.

104. Verberk, W. 2011. Explaining general patterns in species abundance and distributions. Nature Education Knowledge, 3(10): 38.

105. Vetaas, O.R. and Grytnes, J.A., 2002. Distribution of vascular plant species richness and endemic richness along the Himalayan elevation gradient in Nepal. Global Ecology and Biogeography, 11: 291-301.

106. Waterhouse, F.L., Mather, M.H. and Seip, D., 2002. Distribution and abundance of birds relative to elevation and biogeoclimatic zones in coastel old-growth forests in southern British Columbia. J. Ecosys. Manage., 2(2): 1-13.

107. Watson, J.E.M., Whittaker, R.J. and Dawson, T.P., 2004. Habitat structure and proximity to forest edge affect the abundance and distribution of forestdependent birds in tropical coastal forests of southern Madgascar. Biological Conservation, 120: 311-327.

108. Wesolowski, T., Mitrus, C., Czeszczwik, D. and Rowinski, P., 2010. Breeding bird dynamics in a primeval temperate forest over thirty-five years: variation and stability in the changing world. Acta Orn., 45: 209-232.

109. Wright, S.J., 2010. The future of tropical forests. Ann. N. Y. Acad. Sci., 1195: 1-27.

110. Wu, Y.J., Colwell, R.K., Rahbek, C., Zhang, C.L., Quan, Q., Wang, C.K. and Lei, F.M., 2013. Explaining the species richness of birds along a subtropical elevational gradient in Hemgduan Mountains. J. Biogeography, 40: 2310-2323.

111. Yates, C.J., Robinson,T., Wardell-Johnson, G.W., Keppel, G., Hopper, S.D., Scut, A.G. and Byrne, M. 2019. High species diversity and turnover in granite inselberg floras highlight the need for a conservation strategy protecting many outcrops. Ecology and Evolution, 9(13): 7660-7675.

\section{Figures}

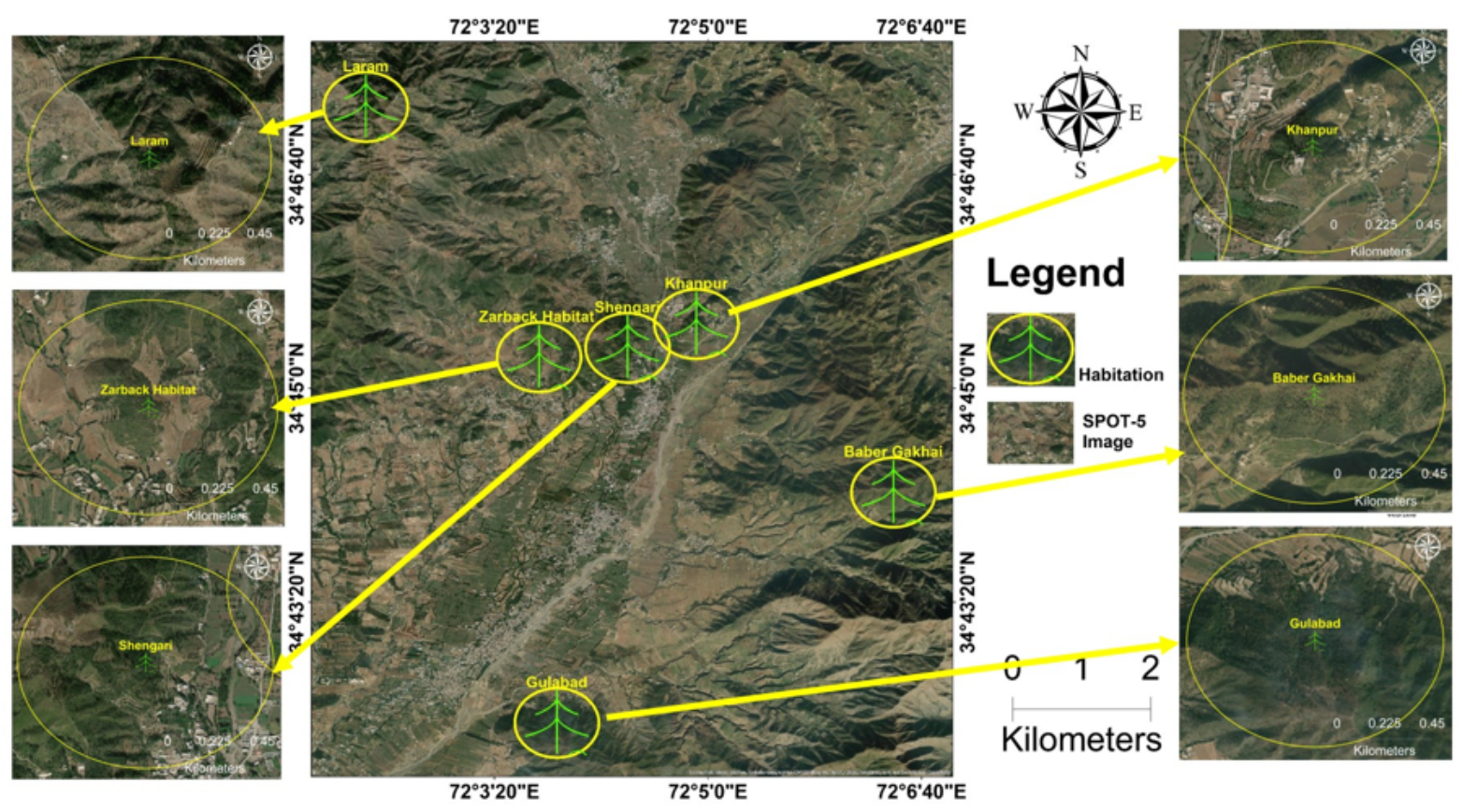

\section{Figure 1}

Map of the study area. Note: The designations employed and the presentation of the material on this map do not imply the expression of any opinion whatsoever on the part of Research Square concerning the legal status of any country, territory, city or area or of its authorities, or concerning the delimitation of its frontiers or boundaries. This map has been provided by the authors. 

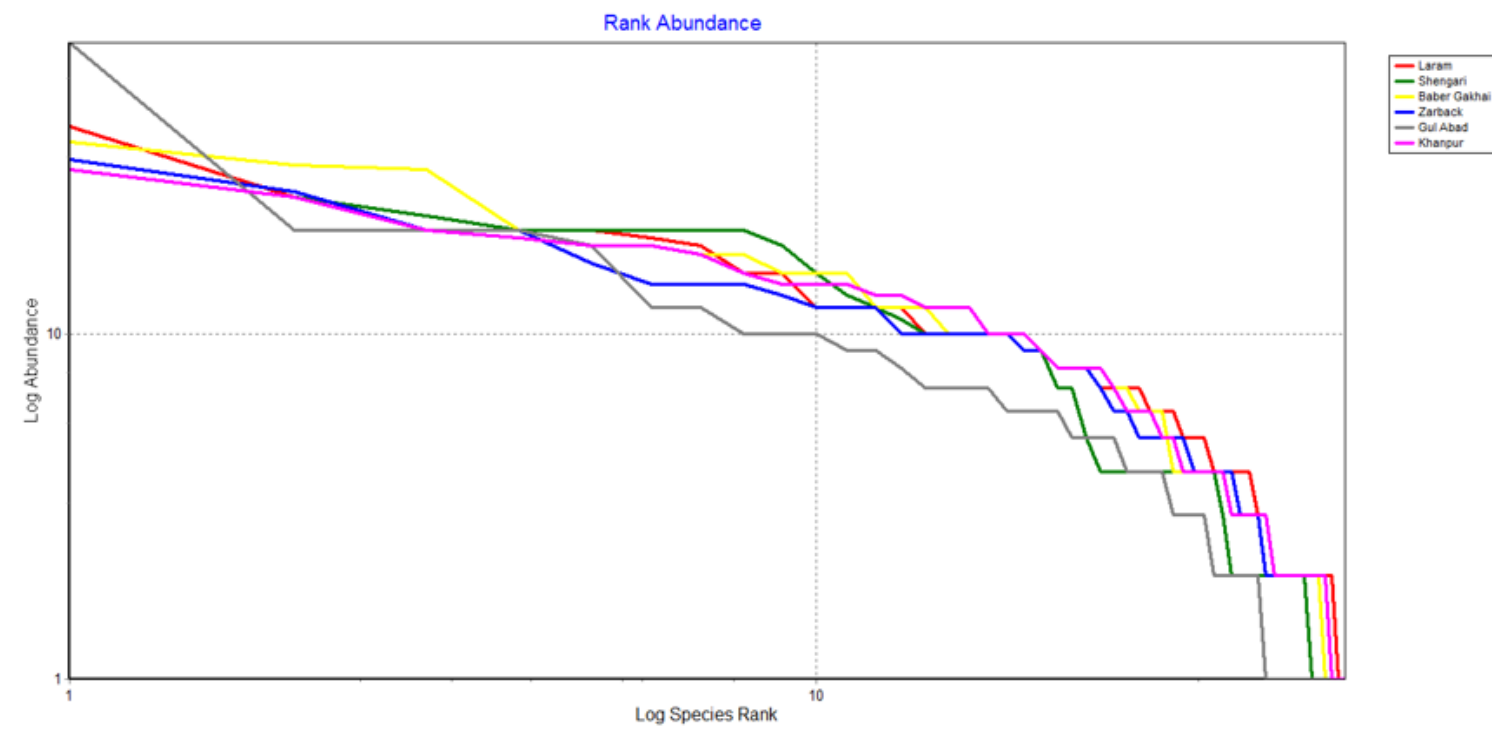

Figure 2

Rank relative abundance curve indicating difference in bird assemblages among habitats 